\title{
Barium and related stars, and their white-dwarf companions
}

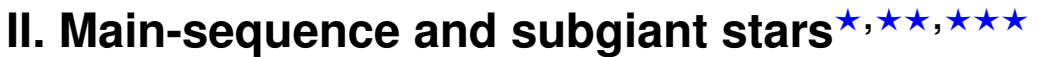

\author{
A. Escorza ${ }^{1,2}$, D. Karinkuzhi ${ }^{2,3}$, A. Jorissen ${ }^{2}$, L. Siess ${ }^{2}$, H. Van Winckel ${ }^{1}$, D. Pourbaix ${ }^{2}$, C. Johnston ${ }^{1}$, \\ B. Miszalski ${ }^{4,5}$, G.-M. Oomen ${ }^{1,6}$, M. Abdul-Masih ${ }^{1}$, H. M. J. Boffin ${ }^{7}$, P. North ${ }^{8}$, R. Manick ${ }^{1,4}$, \\ S. Shetye ${ }^{2,1}$, and J. Mikołajewska ${ }^{9}$
}

\author{
1 Institute of Astronomy, KU Leuven, Celestijnenlaan 200D, 3001 Leuven, Belgium \\ e-mail: ana .escorza@kuleuven . be \\ 2 Institut d'Astronomie et d'Astrophysique, Université Libre de Bruxelles, Boulevard du Triomphe, 1050 Bruxelles, Belgium \\ 3 Department of physics, Jnana Bharathi Campus, Bangalore University, Bangalore 560056, India \\ 4 South African Astronomical Observatory, PO Box 9, Observatory 7935, South Africa \\ 5 Southern African Large Telescope Foundation, PO Box 9, Observatory 7935, South Africa \\ ${ }^{6}$ Department of Astrophysics/IMAPP, Radboud University, PO Box 9010, 6500 GL Nijmegen, The Netherlands \\ 7 ESO, Karl-Schwarzschild-str. 2, 85748 Garching bei München, Germany \\ ${ }^{8}$ Institut de Physique, Laboratoire d'astrophysique, École Polytechnique Fédérale de Lausanne (EPFL), Observatoire, \\ 1290 Versoix, Switzerland \\ 9 N. Copernicus Astronomical Center, Polish Academy of Sciences, Bartycka 18, 00-716 Warsaw, Poland
}

Received 4 March 2019 / Accepted 5 April 2019

\begin{abstract}
Barium (Ba) dwarfs and $\mathrm{CH}$ subgiants are the less evolved analogues of $\mathrm{Ba}$ and $\mathrm{CH}$ giants. They are F- to G-type main-sequence stars polluted with heavy elements by their binary companions when the companion was on the asymptotic giant branch (AGB). This companion is now a white dwarf that in most cases cannot be directly detected. We present a large systematic study of 60 objects classified as Ba dwarfs or $\mathrm{CH}$ subgiants. Combining radial-velocity measurements from HERMES and SALT high-resolution spectra with radial-velocity data from CORAVEL and CORALIE, we determine the orbital parameters of 27 systems. We also derive their masses by comparing their location in the Hertzsprung-Russell diagram with evolutionary models. We confirm that $\mathrm{Ba}$ dwarfs and $\mathrm{CH}$ subgiants are not at different evolutionary stages, and that they have similar metallicities, despite their different names. Additionally, $\mathrm{Ba}$ giants appear significantly more massive than their main-sequence analogues. This is likely due to observational biases against the detection of hotter main-sequence post-mass-transfer objects. Combining our spectroscopic orbits with the HIPPARCos astrometric data, we derive the orbital inclination and the mass of the WD companion for four systems. Since this cannot be done for all systems in our sample yet (but should be possible with upcoming Gaia data releases), we also analyse the mass-function distribution of our binaries. We can model this distribution with very narrow mass distributions for the two components and random orbital orientations on the sky. Finally, based on BINSTAR evolutionary models, we suggest that the orbital evolution of low-mass Ba systems can be affected by a second phase of interactions along the red giant branch of the Ba star, which impact the eccentricities and periods of the giants.
\end{abstract}

Key words. stars: late-type - stars: chemically peculiar - binaries: spectroscopic - techniques: imaging spectroscopy

\footnotetext{
* Radial velocity data are only available at the CDS via anonymous ftp to cdsarc.u-strasbg.fr $(130.79 .128 .5)$ or via http: //cdsarc.u-strasbg.fr/viz-bin/qcat?J/A+A/626/A128

$\star \star$ Based on observations carried out with the Flemish Mercator Telescope at the Spanish Observatorio del Roque de los Muchachos (ORM, La Palma, Spain), the Swiss 1m telescope at the Haute-Provence Observatory (OHP, France), and the $1.54 \mathrm{~m}$ Danish telescope and the Swiss $1.2 \mathrm{~m}$ telescope at the European Southern Observatory (ESO, La Silla, Chile).

$\star \star \star$ Based on observations obtained with the HERMES spectrograph, which is supported by the Fund for Scientific Research of Flanders (FWO), Belgium; the Research Council of KU Leuven, Belgium; the Fonds National de la Recherche Scientifique (F.R.S.-FNRS), Belgium; the Royal Observatory of Belgium; the Observatoire de Genève, Switzerland; and the Thüringer Landessternwarte Tautenburg, Germany.
}

\section{Introduction}

Barium (Ba) stars or Ba II stars, as they were originally named, are G- and K-type giants with peculiarly strong absorption lines of slow-neutron-capture (s)-process elements in their spectra in combination with enhanced $\mathrm{CH}, \mathrm{CN}$, and $\mathrm{C}_{2}$ molecular bands. They were first identified as chemically peculiar by Bidelman \& Keenan (1951), who discussed their distinctive spectroscopic characteristics and particularly stressed the extraordinary strength of the resonance line of ionised barium at $4554 \AA$. The resulting overabundance of barium and other s-process elements on the surface of red giant stars could not be explained from an evolutionary point of view because the s-process of nucleosynthesis takes place in the interiors of asymptotic giant branch (AGB) stars. Barium giants occupy the 
first giant branch (RGB) or the Red Clump (Escorza et al. 2017; and references therein), and are hence not advanced enough in their evolution to produce and dredge up s-process elements.

However, Ba stars are now understood to originate from a binary evolution channel. According to this formation scenario, the carbon and the s-process elements were transferred to the current primary from a more evolved companion when this more evolved companion was in its AGB phase. This implies that the companions of Ba stars are white dwarfs (WDs). The presence of these WD companions has since been indirectly and directly supported. For example, Webbink (1986), McClure \& Woodsworth (1990), Jorissen et al. (1998, 2019), and Merle et al. (2016) have found that the mass-function distribution of Ba giants is consistent with a narrow distribution of companion masses peaking at $0.6 M_{\odot}$. Additionally, Böhm-Vitense et al. $(1984,2000)$ and Gray et al. (2011), among others, have detected UV excess flux from some Ba star systems, which can be attributable to a WD companion.

The orbital properties of Ba giants ( $\mathrm{gBa}$ ) have been intensively and systematically studied (e.g. McClure 1984; McClure \& Woodsworth 1990; Udry et al. 1998a; Jorissen et al. 1998, 2016, 2019) since they are a prototypical family of post-masstransfer low- or intermediate-mass binary systems. A remaining long-standing problem concerning these objects is that binary evolutionary models cannot account for their observed orbital properties. Distributions of observed periods and eccentricities, and abundances of s-process elements are not reproduced well by these models (e.g. Pols et al. 2003; Bonačić Marinović et al. 2008; Izzard et al. 2010). This is a common problem among post-interaction binary systems, such as post-AGB stars (e.g. Oomen et al. 2018), CH stars (e.g. McClure \& Woodsworth 1990), carbon-enhanced metal-poor stars (e.g. Izzard et al. 2010; Jorissen et al. 2016; Abate et al. 2018), blue stragglers (e.g. Mathieu et al. 2015), symbiotic stars (e.g. Mikołajewska 2012), and subdwarf B-type binaries (e.g. Vos et al. 2017).

$\mathrm{CH}$ stars are closely related to $\mathrm{Ba}$ stars and are seen as their Population II analogues. They are also evolved stars with similar enhancement of s-process elements and strong $\mathrm{CH}$ molecular bands. Overall, however, they have weaker metal lines because they belong to an older and more metal-deficient population. They were identified as chemically peculiar for the first time by Keenan (1942).

At lower luminosities dwarf barium ( $\mathrm{dBa}$ ) stars have been much less intensively studied. They are thought to be the less evolved analogues of barium giants as they are F- or G-type main-sequence stars with the same enhancement of carbon and heavy elements (North \& Duquennoy 1991; North \& Lanz 1991; Jorissen et al. 1992; North et al. 1994, 2000). Similarly, subgiant $\mathrm{CH}$ stars $(\mathrm{sgCH})$, which were first identified by Bond (1974), are the low-metallicity counterparts of barium dwarfs and historically thought to be slightly more evolved. However, making the distinction between these two subclasses is not straightforward. We recently suggested that there is no separation between subgiant $\mathrm{CH}$ stars and dwarf Ba stars in the Hertzsprung-Russell diagram (HRD), contrary to what is implied by their designations (Escorza et al. 2017).

In this paper, we focus on the stellar and binary properties of $\mathrm{Ba}$ dwarfs and $\mathrm{CH}$ subgiants. We combine new radialvelocity measurements obtained from high-resolution spectra with archival radial-velocity data and determine the orbital parameters of a sample of these objects. In this way, we significantly increase the number of known orbits of main-sequence and subgiant $\mathrm{Ba}$ and $\mathrm{CH}$ stars, and we can compare these populations with their evolved analogues. We describe the stel- lar sample and the data sets in Sect. 2 and our method to determine the best orbital solution in Sect. 3. The results are presented in Sect. 4, where we also describe some individual targets that deserve special attention. Additionally, in Sect. 5, we use stellar parameters derived from high-resolution spectra, and the distances derived by Bailer-Jones et al. (2018) from Gaia DR2 parallaxes (Lindegren et al. 2018) to plot our targets on a HRD and derive their masses by comparing their location with STAREVOL evolutionary models (Siess et al. 2000; Siess \& Arnould 2008). Finally in Sect. 6, we discuss the stellar and orbital properties of our main-sequence stars and compare them with a well-studied sample of Ba giants. We also analyse the derived binary mass-functions to obtain information about the WD companion. For four of our objects, we derive absolute WD masses using orbital inclinations obtained by reprocessing Hipparcos astrometric data. Finally, we use BINSTAR binary evolution models (Siess et al. 2013) to explore the evolutionary link between our main-sequence and subgiants stars and the better known $\mathrm{Ba}$ and $\mathrm{CH}$ giants.

\section{Sample overview and data description}

In 1984, a monitoring campaign of barium and related stars was initiated with the CORrelation-RAdial-VELocities (CORAVEL) spectrometers (Baranne et al. 1979). Some results of this programme were presented in Jorissen \& Mayor (1988), Jorissen et al. (1998), and Udry et al. (1998a). Due to the long periods of some of these binaries, the CORAVEL monitoring was not long enough to derive the orbital parameters of all the objects in the sample. Several years after the CORAVEL programme was interrupted, the monitoring of these families of binaries was resumed with the High-Efficiency and high-Resolution Mercator Echelle Spectrograph (HERMES; Raskin et al. 2011). Combining the older CORAVEL radial-velocity measurements with the more recent HERMES data, the total time coverage amounts to more than 30 years, and a full orbital cycle can now be covered for some long-period binaries for the first time. The combination of these two data sets has proven successful in past studies (e.g. Jorissen et al. 2016; Oomen et al. 2018). Additionally, thanks to the higher accuracy of the HERMES radial velocities, systems with much lower amplitude radial-velocity variations could also be revealed. The HERMES spectrograph is limited to observing objects with declination $\gtrsim-30^{\circ}$, thus the southern objects in our sample were observed with the $11 \mathrm{~m}$ Southern African Large Telescope (SALT; Buckley et al. 2006; O’Donoghue et al. 2006).

\subsection{Radial-velocity measurements with CORAVEL and CORALIE}

The monitoring campaign of Ba stars started with data from the two CORAVEL spectrometers: one installed on the $1 \mathrm{~m}$ Swiss telescope at the Haute-Provence Observatory for the northern sky and the other installed on the $1.54 \mathrm{~m}$ Danish telescope at ESO-La Silla (Chile) for the southern sky (Baranne et al. 1979). The CORAVEL radial velocities were obtained by crosscorrelation and Gaussian-fitting techniques on the data obtained via the instrument, which has a hardware spectral mask based on a spectrum of Arcturus (K1.5III; see Baranne et al. 1979; Duquennoy et al. 1991, or Udry et al. 1998a for more details).

The radial-velocity standard stars used to fix the zero-point of the HERMES spectrograph are tied to the ELODIE velocity system (Udry et al. 1999). However, some of our CORAVEL radial velocities are from before 1999 and are tied to an old 


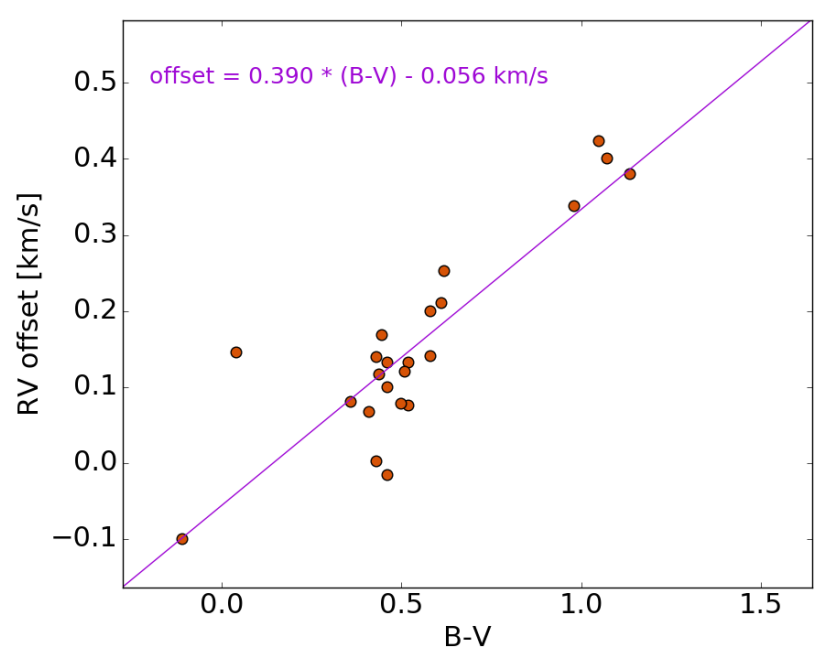

Fig. 1. Relation between the $B-V$ index of the stars and the radialvelocity offset applied on the old CORAVEL data to make it compatible with data sets tied to the new ELODIE system.

velocity system, previous to the ELODIE system. Hence, a zeropoint offset needs to be applied to these old measurements in order to convert them to the ELODIE system and combine them with HERMES data. Fortunately, we were able to access reprocessed pre-1999 CORAVEL data for some objects and to use the difference between the reprocessed and the uncorrected data to define an offset for the rest of the stars. The zero-point offset depends on the $B-V$ index (Udry et al. 1999); Fig. 1 shows the relation that we obtained and applied to our other targets. We note that the scatter is not larger than the average CORAVEL error bar $\left(\sim 0.3 \mathrm{~km} \mathrm{~s}^{-1}\right)$.

Additionally, a few objects have been observed with the CORALIE spectrograph installed on the Swiss 1.2-metre Leonhard Euler Telescope at ESO-La Silla (Chile) after CORAVEL was decommissioned in 1998. For one target, HD 48565, we also have four radial-velocity measurements from ELODIE, an echelle spectrograph installed at the Haute-Provence Observatory $1.93 \mathrm{~m}$ reflector in south-eastern France.

\subsection{Radial-velocity monitoring with the HERMES spectrograph}

The targets with declinations higher than $-30^{\circ}$ have been observed with HERMES, the state-of-the-art fiber echelle spectrograph mounted on the $1.2 \mathrm{~m}$ Flemish Mercator telescope at the Spanish Observatorio del Roque de los Muchachos in La Palma. These targets are part of the long-term monitoring programme of binaries (Gorlova et al. 2013) carried out with this instrument thanks to the agreement reached by the consortium partners (KU Leuven, Université libre de Bruxelles, Royal Observatory of Belgium, Observatoire de Genève, and Thüringer Landessternwarte Tautenburg). As shown by many studies, scientific advancement in the study of evolved wide binaries is only made possible by regular long time-base monitoring, such as that guaranteed by HERMES (e.g. Van Winckel et al. 2010; Vos et al. 2015; Manick et al. 2017; Oomen et al. 2018).

The instrument, which is fully described in Raskin et al. (2011), covers a spectral range from $380 \mathrm{~nm}$ to $900 \mathrm{~nm}$ and has a spectral resolution of about 85000 for the high-resolution science fibre. The temperature-controlled environment ensures the long-term stability of the radial velocities. The radial velocities are obtained by performing a Gaussian fit on the cross- correlation function (CCF) obtained with a spectral mask matching the spectral type of the target stars. In our case, depending on the spectral type reported in the literature, we used the F0 or G2 masks in orders $55-74(478 \mathrm{~nm}-653 \mathrm{~nm})$, as they give the best compromise between absence of telluric lines and maximum signal-to-noise ratio for F-G-K type stars.

Crucial to obtaining accurate radial velocities is the wavelength calibration of HERMES. Two lamps (ThAr and Ne) are used to obtain several wavelength reference exposures over the night to correct for the possible pressure drifts (see Fig. 9 of Raskin et al. 2011). Additionally, the long-term accuracy can be estimated from the stability of RV standard stars (from the list of Udry et al. 1999) monitored along with the science targets. The distribution of the radial-velocity standards gives a $1 \sigma$ dispersion of $63 \mathrm{~m} \mathrm{~s}^{-1}$ (see Fig. 10 of Raskin \& Van Winckel 2014), which we adopt as the typical uncertainty on the radial velocities over the long term.

\subsection{Radial velocities with SALT HRS}

We complemented our data sets with spectra obtained with the SALT High Resolution Spectrograph (HRS; Bramall et al. 2010, 2012; Crause et al. 2014). The targets were included as poorweather targets in the SALT programme 2017-1-MLT-010 (PI: Miszalski; see e.g. Miszalski et al. 2018a,b). The HRS is a dual-beam, fibre-fed echelle spectrograph enclosed in a vacuum tank located in an insulated, temperature controlled room of the $11 \mathrm{~m}$ Southern African Large Telescope (Buckley et al. 2006; O'Donoghue et al. 2006). The medium-resolution (MR) mode of HRS was used, and the spectra covered a wavelength range from $370 \mathrm{~nm}$ to $890 \mathrm{~nm}$ with resolving powers $R=\lambda / \Delta \lambda$ of 43000 and 40000 for the blue and red arms, respectively. Regular bias, ThAr arc and quartz lamp flat-field calibrations are taken. The basic data products (Crawford et al. 2010) were reduced with the MIDAS pipeline developed by Kniazev et al. (2016), which is based on the ECHELLE (Ballester et al. 1992) and FEROS (Stahl et al. 1999) packages. Heliocentric corrections were applied to the data using VELSET of the RVSAO package (Kurtz \& Mink 1998). We used the same spectral masks that we used to derive the radial velocities for the HERMES spectra by means of a Gaussian fit to the CCF.

To make sure we could combine our data sets meaningfully, in 2018 we observed a radial-velocity standard star with both SALT HRS and HERMES. We chose HD 156365 because its declination $\left(\delta \sim-24^{\circ}\right)$ makes it observable with both instruments. In the catalogue of radial-velocity standard stars for Gaia (Soubiran et al. 2013), the radial-velocity measurements of HD 156365 range from -13.06 to $-13.11 \mathrm{~km} \mathrm{~s}^{-1}$. We obtained $\mathrm{RV}_{\text {SALTHRS }}=-13.30 \pm 0.17 \mathrm{~km} \mathrm{~s}^{-1}$ and $\mathrm{RV}_{\mathrm{HER} 18}=$ $-12.98 \pm 0.07 \mathrm{~km} \mathrm{~s}^{-1}$. In the data archive of HERMES, there was another spectrum of this star from 2013 for which we obtained $\mathrm{RV}_{\mathrm{HER} 13}=-13.08 \pm 0.07 \mathrm{~km} \mathrm{~s}^{-1}$. We conclude that this limited experiment shows a slight offset of about $260 \mathrm{~m} \mathrm{~s}^{-1}$ between SALT HRS and HERMES. However, as the number of HERMES and CORAVEL observations is so large, we decided not to apply any systematic offset to the SALT-HRS data.

\subsection{Stellar sample}

Our sample includes 60 stars that have been identified as $\mathrm{Ba}$ dwarfs or $\mathrm{CH}$ subgiants in past studies or as suspected candidates. An important fraction of the present sample was already included in North et al. (2000), where the authors used radial velocities from CORAVEL and from the high-resolution Coudé Echelle Spectrometer (CES) installed at the ESO CAT 
$1.4 \mathrm{~m}$ telescope. We complement their data set with HERMES, CORALIE, and SALT data. Several new targets were also added to the sample. The following objects were monitored:

- Thirteen confirmed dBa stars from North et al. (1994): $\mathrm{BD}+18^{\circ} 5215$, HD 15306, HD 48565, HD 76225, HD 92545, HD 106191， HD 107574, HD 113402, HD 147609, HD 188985, HD 202400, HD 221531, and HD 222349;

- Seven targets from Houk \& Cowley (1975). Five of them (HD 18853, HD 24864, HD 26455, HD 31732, and HD 69578) were reported to have "strong Sr 4077", as confirmed in North et al. (2000). HD 9529 presents a small overabundance of yttrium, and BD-18 255 was later classified as a CH subgiant by Luck \& Bond (1991) and again as a dBa star by North (1995);

- Two dBa stars from the catalogue of Lü et al. (1983): HD 50264 and HD 87080. They were later classified as CH subgiants by Pereira \& Junqueira (2003);

- Three gBa stars from the catalogue of Lü et al. (1983), which seem to be either main-sequence or subgiant stars in our HRD (see Sect. 7): HD 22589, HD 120620, and HD 216219. Their orbital elements were already determined by Udry et al. (1998a,b). However, since we are now able to add new radial velocity data points, we refit their orbits and included them as barium dwarfs in our sample;

- One dBa star from Tomkin et al. (1989): HD 2454;

- One dBa star from Houk (1978): HD 109490;

- Two dBa stars identified by Gray et al. (2011): HD 34654 and HD 114520;

- Nine mild Ba dwarfs from Edvardsson et al. (1993) with marginal overabundance of s-process elements with respect to iron, typically of the order of $[\mathrm{s} / \mathrm{Fe}] \approx 0.2$ : HD 6434 , HD 13555, HD 35296, HD 60532, HD 82328, HD 95241, HD 98991, HD 124850, HD 220117;

- Ten objects listed as suspected Ba dwarfs in Table 2 of Lü (1991): HD 101581, HD 103840, HD 104342, HD 105671, HD 117288, HD 146800, HD 170149, HD 177996, HD 205156, HD 219899. One of them, HD 177996, has been reported as an active pre-main-sequence star by Henry et al. (1996) and as a double-lined spectroscopic binary (SB2) by Soderblom et al. (1998). We confirm the latter classification from the SALT-HRS data;

- Eleven CH subgiants from Luck \& Bond (1991):

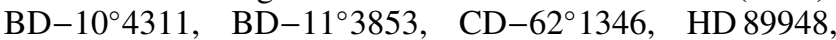
HD 123585, HD 127392, HD 141804, HD 150862, HD 182274, HD 207585, and HD 224621;

- One CH subgiant from McClure \& Woodsworth (1990): HD 130255.

To our knowledge, with a sample of 60 objects, this is the largest systematic radial-velocity survey of dwarf and subgiant $\mathrm{Ba}$ and $\mathrm{CH}$ stars. Table A.1 lists information about the stars and about the available observations.

\section{Orbital analysis}

The orbital parameters, i.e. the period $(P)$, the eccentricity $(e)$, the time of periastron passage $\left(T_{0}\right)$, the longitude of periastron $(\omega)$, the velocity semi-amplitude $\left(K_{1}\right)$, and the systemic velocity of the system $(\gamma)$, were determined for each target by fitting a Keplerian orbit to its radial-velocity curve (see Table 1). We obtained an initial model by applying a minimisation method using the dominant frequency found in the periodogram of the radial-velocity data. We then computed a second model by means of an iterative non-linear least-squares minimisation procedure, which uses as convergence criterion the comparison of consecutive variance reductions. Additionally, an extra model was fitted to the data for which we imposed a circular orbit. For each target, we applied the Lucy \& Sweeney test (Lucy \& Sweeney 1971) to avoid the determination of spurious eccentricities for truly circular orbits. This test compares the sum of the squared residuals of the best-fitting eccentric orbit (indicated with the subscript ecc) with those of a circular fit (indicated with the subscript $\operatorname{circ}$ ) through the determination of the parameter $p$ as indicated in Eq. (1)

$p=\left(\frac{\Sigma(o-c)_{\mathrm{ecc}}^{2}}{\Sigma(o-c)_{\mathrm{circ}}^{2}}\right)^{(n-m) / 2}$

where $n$ is the total number of observations and $m=6$ is the number of free parameters in an eccentric fit. Lucy \& Sweeney (1971) argued that only when $p<0.05$ can the orbit be considered significantly eccentric. Once the mentioned parameters were determined from the radial-velocity fitting, we were also able to compute the mass function $f(m)$ and the apparent orbital separation $a_{1} \sin i$, which depend on $P, e$, and $K_{1}$. These results are also included in Table 1.

We used a Monte Carlo method to determine the uncertainties of the orbital parameters. We generated $1000 \mathrm{RV}$ curves for each fitted binary by randomly sampling new RV data normally distributed around the best-fitting model and within the standard deviation of the residuals of the fit. The $1 \sigma$ uncertainties were chosen as the standard deviation of each parameter after fitting these $1000 \mathrm{RV}$ curves. We note that circular orbits have a one-sided uncertainty and do not have an argument of periastron. The time of periastron passage is replaced by the epoch of maximum velocity.

Finally, due to the better quality of the HERMES data (see the error bars of the two data sets in Fig. B.2 as an example), whenever an orbit could be fully covered with HERMES radial velocities, we decided to determine the orbital parameters using only HERMES data. Then data points from other instruments were overplotted to confirm the fit. This was the case for BD-18 255 , HD 107574, HD 147609, and HD 221531 and it was tested that the orbital parameters remain the same within the errors when the other data sets were included. This can also be seen as a confirmation of the offset applied to CORAVEL data and of the potential of including SALT HRS to determine the orbital parameters of the long and uncovered southern Ba star systems.

\section{Results}

Our analysis confirmed binarity for 40 out of our 60 objects, and we constrained 27 spectroscopic orbits. Table 1 lists the orbital elements derived in this work. Figures with the best-fitting models and the residuals of the fit are included in Appendix B.

\subsection{Single-lined spectroscopic binaries}

We derived orbital elements for 25 single-line spectroscopic binaries (SB1). Some of these binaries were already discussed by North et al. (2000), but their orbital elements were never published. However, five of them have spectroscopic orbits published and included in the ninth catalogue of spectroscopic binary orbits (SB9; Pourbaix et al. 2004). As mentioned before, the orbits of the three stars flagged as $\mathrm{gBa}$ stars in the literature, HD 22589, HD 120620, and HD 216219, were determined by Udry et al. (1998a,b). Our new orbital elements are in agreement with theirs within uncertainties. In addition, Griffin (2018) determined orbital elements for HD 34654 from 
Table 1. Orbital parameters (i.e. period, $P$; eccentricity, $e$; time of periastron passage, $T_{0}$; longitude of periastron, $\omega$; velocity semi-amplitude, $K_{1}$; and the systemic velocity of the system, $\gamma$ ) derived in this study.

\begin{tabular}{|c|c|c|c|c|c|c|c|c|c|c|}
\hline Name & Type & Period [d] & $e$ & $T_{0}[\mathrm{HJD}]$ & $\omega\left[^{\circ}\right]$ & $K_{1}\left[\mathrm{~km} \mathrm{~s}^{-1}\right]$ & $\gamma\left[\mathrm{km} \mathrm{s}^{-1}\right]$ & $f(m)\left[M_{\odot}\right]$ & $a_{1} \sin i[\mathrm{AU}]$ & $\begin{array}{l}\sigma(O-C) \\
{\left[\mathrm{km} \mathrm{s}^{-1}\right]}\end{array}$ \\
\hline BD- $10^{\circ} 4311$ & $\mathrm{sgCH}$ & $4865 \pm 20$ & $0.075 \pm 0.019$ & $2451183 \pm 2500$ & $157 \pm 17$ & $5.29 \pm 0.11$ & $50.95 \pm 0.08$ & $0.074 \pm 0.005$ & $2.36 \pm 0.05$ & 0.48 \\
\hline BD-1 $18^{\circ} 255$ & $\mathrm{dBa}$ & $1585 \pm 6$ & $0.075 \pm 0.011$ & $2458136 \pm 730$ & $124 \pm 8$ & $7.25 \pm 0.07$ & $34.65 \pm 0.05$ & $0.0620 \pm 0.0017$ & $1.053 \pm 0.010$ & 0.28 \\
\hline $\mathrm{BD}+18^{\circ} 5215$ & $\mathrm{dBa}$ & $1516 \pm 11$ & $0.13 \pm 0.06$ & $2447317 \pm 800$ & $72 \pm 28$ & $3.09 \pm 0.16$ & $-30.19 \pm 0.10$ & $0.0045 \pm 0.0007$ & $0.43 \pm 0.02$ & 0.53 \\
\hline HD 15306 & $\mathrm{dBa}$ & $8383 \pm 48$ & $0.20 \pm 0.03$ & $2448335 \pm 2400$ & $140 \pm 16$ & $3.67 \pm 0.14$ & $46.82 \pm 0.14$ & $0.040 \pm 0.005$ & $2.77 \pm 0.11$ & 0.46 \\
\hline HD 22589 & $\mathrm{gBa}$ & $5573 \pm 33$ & $0.232 \pm 0.016$ & $2448567 \pm 2800$ & $333 \pm 4$ & $1.90 \pm 0.03$ & $-27.51 \pm 0.03$ & $0.00367 \pm 0.00018$ & $0.949 \pm 0.017$ & 0.13 \\
\hline HD 24864 & $\mathrm{dBa}$ & $430 \pm 3$ & $0.0+0.05$ & $2449475 \pm 600$ & - \pm- & $7.6 \pm 0.8$ & $13.1 \pm 0.3$ & $0.019 \pm 0.005$ & $0.30 \pm 0.03$ & 0.98 \\
\hline HD 34654 & $\mathrm{dBa}$ & $975.6 \pm 0.6$ & $0.110 \pm 0.007$ & $2457161 \pm 130$ & $326 \pm 2$ & $8.90 \pm 0.04$ & $-6.89 \pm 0.02$ & $0.0699 \pm 0.0011$ & $0.793 \pm 0.004$ & 0.05 \\
\hline HD 50264 & $\mathrm{dBa}$ & $909.9 \pm 1.2$ & $0.091 \pm 0.03$ & $2449541 \pm 60$ & $230 \pm 15$ & $9.52 \pm 0.14$ & $63.27 \pm 0.19$ & $0.080 \pm 0.003$ & $0.793 \pm 0.012$ & 0.35 \\
\hline HD 76225 & $\mathrm{dBa}$ & $2410 \pm 2$ & $0.098 \pm 0.005$ & $2451159 \pm 400$ & $267 \pm 3$ & $6.11 \pm 0.04$ & $30.34 \pm 0.02$ & $0.0561 \pm 0.0010$ & $1.347 \pm 0.008$ & 0.09 \\
\hline HD 87080 & $\mathrm{sgCH}$ & $274.3 \pm 1.9$ & $0.161 \pm 0.007$ & $2448921 \pm 20$ & $128 \pm 2$ & $12.17 \pm 0.07$ & $-2.94 \pm 0.05$ & $0.0492 \pm 0.0008$ & $0.3029 \pm 0.0017$ & 0.14 \\
\hline HD 89948 & $\mathrm{sgCH}$ & $673 \pm 3$ & $0.12 \pm 0.03$ & $2448225 \pm 300$ & $135 \pm 7$ & $9.9 \pm 0.2$ & $14.91 \pm 0.14$ & $0.065 \pm 0.005$ & $0.606 \pm 0.016$ & 0.32 \\
\hline HD 95241 & $\mathrm{dBa}$ & $5244 \pm 56$ & $0.82 \pm 0.02$ & $2450499 \pm 2000$ & $112 \pm 3$ & $4.5 \pm 0.2$ & $-7.98 \pm 0.05$ & $0.0091 \pm 0.0013$ & $1.23 \pm 0.05$ & 0.2 \\
\hline HD 98991 & $\mathrm{dBa}$ & $2836 \pm 99$ & $0.317 \pm 0.008$ & $2455888 \pm 1400$ & $25 \pm 20$ & $4.70 \pm 0.08$ & $14.06 \pm 0.04$ & $0.0259 \pm 0.0007$ & $1.16 \pm 0.03$ & 0.11 \\
\hline HD 106191 & $\mathrm{dBa}$ & $1314 \pm 13$ & $0.15 \pm 0.10$ & $2446527 \pm 1300$ & $120 \pm 34$ & $7.1 \pm 0.5$ & $-3.3 \pm 0.2$ & $0.048 \pm 0.008$ & $0.85 \pm 0.07$ & 0.58 \\
\hline HD 107574 & $\mathrm{dBa}$ & $1384 \pm 2$ & $0.084 \pm 0.004$ & $2457910 \pm 160$ & $218 \pm 4$ & $2.238 \pm 0.009$ & $-28.579 \pm 0.008$ & $0.00159 \pm 0.00002$ & $0.284 \pm 0.002$ & 0.05 \\
\hline HD 120620 & $\mathrm{gBa}$ & $217.2 \pm 1.0$ & $0.0+0.05$ & $2456980 \pm 100$ & - \pm- & $14.02 \pm 0.16$ & $33.56 \pm 0.13$ & $0.062 \pm 0.002$ & $0.280 \pm 0.004$ & 0.401 \\
\hline HD 123585 & $\mathrm{sgCH}$ & $459.4 \pm 1.5$ & $0.0+0.05$ & $2448419 \pm 500$ & - \pm- & $11.7 \pm 0.2$ & $25.49 \pm 0.17$ & $0.077 \pm 0.004$ & $0.496 \pm 0.008$ & 0.49 \\
\hline HD 127392 & $\mathrm{sgCH}$ & $1508 \pm 2$ & $0.093 \pm 0.010$ & $2448739 \pm 700$ & $1711 \pm 10$ & $8.25 \pm 0.09$ & $-64.21 \pm 0.07$ & $0.087 \pm 0.003$ & $1.14 \pm 0.02$ & 0.17 \\
\hline HD 141804 & $\mathrm{sgCH}$ & $2652 \pm 95$ & $0.0+0.05$ & $2449979 \pm 1500$ & - \pm- & $6.28 \pm 0.16$ & $-49.3 \pm 0.2$ & $0.068 \pm 0.007$ & $1.531 \pm 0.09$ & 0.20 \\
\hline HD 147609 & $\mathrm{dBa}$ & $1146.2 \pm 1.5$ & $0.058 \pm 0.005$ & $2455716 \pm 130$ & $123 \pm 5$ & $2.974 \pm 0.016$ & $-16.277 \pm 0.009$ & $0.00310 \pm 0.00005$ & $0.3128 \pm 0.0016$ & 0.057 \\
\hline HD 150862 & $\mathrm{sgCH}$ & $291 \pm 4$ & $0.28 \pm 0.09$ & $2448733 \pm 100$ & $299 \pm 21$ & $12 \pm 2$ & $-82.1 \pm 0.7$ & $0.045 \pm 0.003$ & $0.305 \pm 0.009$ & 0.15 \\
\hline HD 182274 & $\mathrm{sgCH}$ & $8231 \pm 54$ & $0.0+0.05$ & $2451522 \pm 3000$ & - \pm- & $2.99 \pm 0.16$ & $-16.34 \pm 0.19$ & $0.0227 \pm 0.005$ & $2.26 \pm 0.12$ & 0.24 \\
\hline HD 207585 & $\mathrm{sgCH}$ & $672 \pm 2$ & $0.0+0.05$ & $2452172 \pm 500$ & - \pm- & $10.4 \pm 0.3$ & $-62.3 \pm 0.2$ & $0.078 \pm 0.007$ & $0.641 \pm 0.018$ & 0.73 \\
\hline HD 216219 & $\mathrm{gBa}$ & $4117 \pm 122$ & $0.0+0.05$ & $2456272 \pm 2000$ & - \pm- & $2.98 \pm 0.10$ & $-6.82 \pm 0.10$ & $0.0113 \pm 0.0013$ & $1.13 \pm 0.05$ & 0.383 \\
\hline HD 221531 & $\mathrm{dBa}$ & $1399 \pm 3$ & $0.163 \pm 0.007$ & $2456940 \pm 81$ & $184 \pm 4$ & $6.44 \pm 0.04$ & $2.82 \pm 0.02$ & $0.0372 \pm 0.0007$ & $0.818 \pm 0.006$ & 0.14 \\
\hline Name & Type & Period [d] & $e$ & $T_{0}[\mathrm{HJD}]$ & $\omega\left[^{\circ}\right]$ & $K_{i}\left[\mathrm{~km} \mathrm{~s}^{-1}\right]$ & $\gamma\left[\mathrm{km} \mathrm{s}^{-1}\right]$ & $f(m)\left[M_{\odot}\right]$ & $a_{i} \sin i[\mathrm{AU}]$ & $\begin{array}{l}\sigma(O-C) \\
{\left[\mathrm{km} \mathrm{s}^{-1}\right]}\end{array}$ \\
\hline HD 48565 in & Trip. & $73.344 \pm 0.013$ & $0.220 \pm 0.007$ & $2446943 \pm 2$ & $214 \pm 3$ & $9.17 \pm 0.06$ & - & $0.00542 \pm 0.00010$ & $0.0603 \pm 0.0004$ & 1.41 \\
\hline HD $48565_{\text {out }}$ & Trip. & $10531 \pm 210$ & $0.37 \pm 0.06$ & $2464195 \pm 120$ & $222 \pm 5$ & $3.9 \pm 0.2$ & $-21.01 \pm 0.12^{(1)}$ & $0.053 \pm 0.003$ & $3.5 \pm 0.3$ & 0.31 \\
\hline HD $114520 \mathrm{a}$ & SB2 & $437.94 \pm 0.18$ & $0.523 \pm 0.003$ & $2456152 \pm 2$ & $249.7 \pm 0.5$ & $12.41 \pm 0.06$ & $-9.07 \pm 0.03$ & $0.054 \pm 0.002$ & $0.426 \pm 0.005$ & 0.11 \\
\hline HD $114520 \mathrm{~b}$ & SB2 & $"$ & $=4$ & 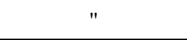 & " & $-17.02 \pm 0.12$ & $"$ & & $-0.138 \pm 0.015$ & 0.75 \\
\hline
\end{tabular}

Notes. The mass function $f(m)$ and the apparent orbital separation $a_{1} \sin i$, which depend on $P, e$, and $K_{1}$, are also included. The last column shows the standard deviation of the residuals of the fit. In the upper part of the table, we list the 25 dwarf and subgiant $\mathrm{Ba}$ and $\mathrm{CH}$ stars that are in SB1 systems. In the bottom part, we include the orbital parameters of the inner and outer orbits of the confirmed triple system, HD 48565 (see Sect. 4.2), and the parameters of the constrained Ba SB2 system, HD 114520 (see Sect. 4.3).

photoelectric radial velocities, and Willmarth et al. (2016) determined orbital elements for HD 95241. Our results are also in good agreement with theirs.

In the following subsections, we focus on some individual systems with remarkable properties.

\section{2. $H D 48565$}

HD 48565 is an SB1 and the dominant signal of its radialvelocity curve can be best fit by a Keplerian orbit with a period of 73 days and an eccentricity of 0.22 (see upper panel of Figs. 2 and 3). This period was already constrained by North et al. (2000), who also noticed a trend in the residuals and suggested the possible presence of a third star in the system, in a much longer orbit.

Combining our HERMES observations with CORAVEL and ELODIE data, we confirm the presence of a trend in the residuals (see second panel of Fig. 3) and estimate the orbital elements of the outer orbit. We assume that the secondary orbit is only a small perturbation of the primary, so we can fit them independently. The best-fitting model of the residuals, with a period of more than 10500 days and an eccentricity of almost 0.4 is presented in the second panel of Fig. 3. The third panel of this figure shows the modelled radial-velocity curve, which results from combining the two Keplerian orbits, and the fourth panel shows the final residuals. To plot the third and fourth panels, we have accounted for light-time effects (LTE) caused by the

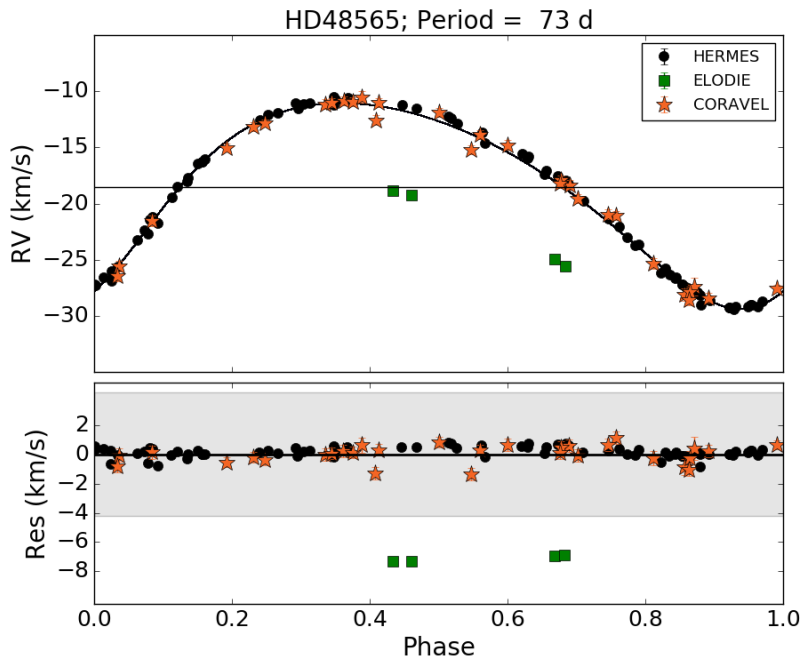

Fig. 2. Phase-folded HERMES and CORAVEL radial velocities and best-fitting orbital model for the short orbit of the triple system HD 48565. Lower panel: observed minus calculated $(O-C)$ residuals of the fit and a shadowed region which corresponds to three times the standard deviation of the residuals.

motion of the inner pair around the centre of mass of the triple. We followed Eq. (1) from Torres \& Stefanik (2000). The orbital parameters of the inner and outer orbits are included in the 


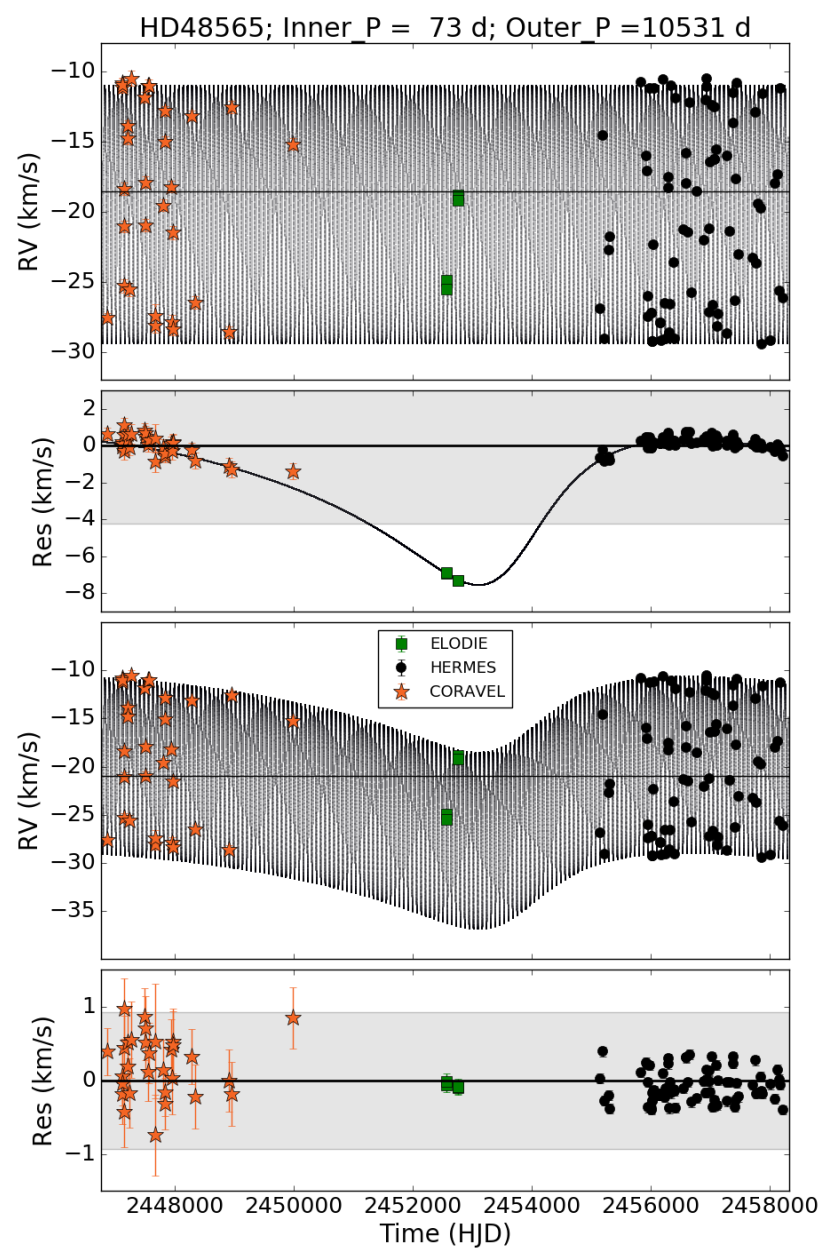

Fig. 3. Same as Fig. 2, but as a function of time. The best-fitting orbital model to the residuals is included in the second panel. The combined fits and residuals are shown in the third and fourth panels, respectively.

bottom part of Table 1 . The systemic velocity of the system was obtained by fixing $\gamma_{\text {in }}$ to zero. Given the ratio of the periods, it is likely that the triple system is dynamically stable (Tokovinin 2014; Toonen et al. 2016).

North et al. (2000) suggested that the inner orbit is too small to have hosted an AGB star, so that the WD must be in the outer orbit while the other two components are less evolved. However, the complexity of the evolution of hierarchical triple systems and the amount of dynamical processes that could have affected HD 48565 make it difficult to describe the history of the system. For example, from Eq. (24) of Toonen et al. 2016, we estimate that Kozai-Lidov cycles in the system are of the order of several thousand years, which are short enough to have an important effect on the evolution of the system. We note that for small relative inclinations, the amplitude of these cycles is zero, so they do not have to exist, but we do not have enough information to know if they do or not. Additionally, the derived mass functions are compatible with the WD being in both orbits. Since we cannot confidently say in which orbit the WD is, we are cautious about including HD 48565 in certain parts of our further analysis.

\section{3. $H D 114520$}

Although its binarity and the strength of its $\mathrm{Sr}$ II line at $4077 \AA$ had been reported before, HD 114520 was first classified as a $\mathrm{Ba}$ dwarf by Gray et al. (2011). From high-resolution spectra, they

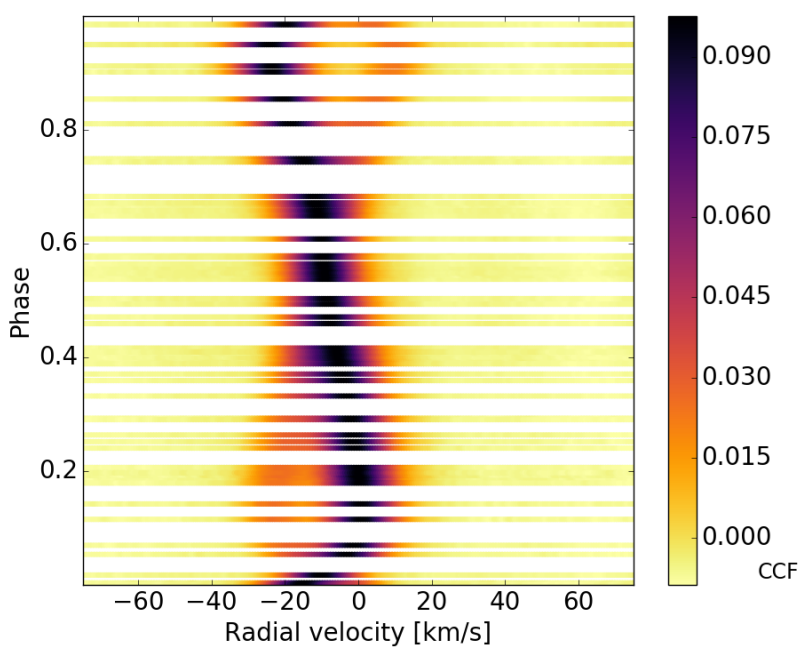

Fig. 4. Intensity map of the CCF profiles of HD 114520 phase-folded using the orbital period of the SB2 $(P=437.8$ days $)$.

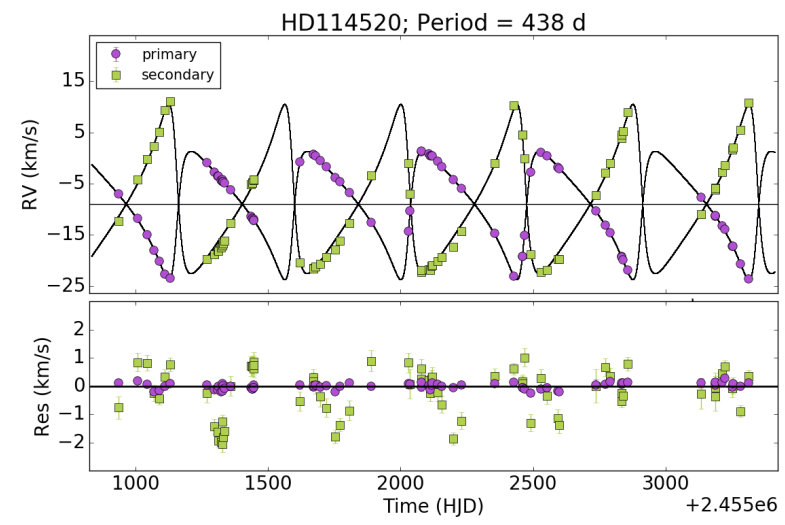

Fig. 5. Radial-velocity curves and orbital fit of the components of the SB2 in HD 114520. Lower panel: $O-C$ residuals of the fit of both components.

reported an overabundance of some s-process elements, and they classified the system as an SB2. They also detected an excess in the UV, which they associated with a WD companion. This means that HD 114520 is also a triple system.

We have 81 high-resolution HERMES spectra of HD 114520 obtained over six years, with which we cover several orbital cycles. In Fig. 4, we show an intensity map with all the available CCF profiles phase-folded with the dominant period found in the data ( 438 days). At given phases, the CCF clearly shows a double peak confirming the SB2 nature of the system. The good orbital coverage and the high eccentricity of the system are also clear from this figure.

We obtained the radial-velocity data of the two components by fitting a double-Gaussian to the CCF profile of each HERMES spectrum. To obtain accurate orbital parameters, we excluded a few data points obtained at phases of zero or almost zero relative radial-velocity between the two stars. Disentangling the two contributions to the CCF at these phases was not easy and the poor quality of the radial-velocity points strongly affected the orbital fit. The best-fitting solution to the two components is shown in Fig. 5 and the derived orbital parameters are included in Table 1 . We can also calculate the mass ratio between the two stars as $q=m_{2} / m_{1}=K_{1} / K_{2}=0.73$. The secondary is probably another main-sequence star of a cooler spectral type. 

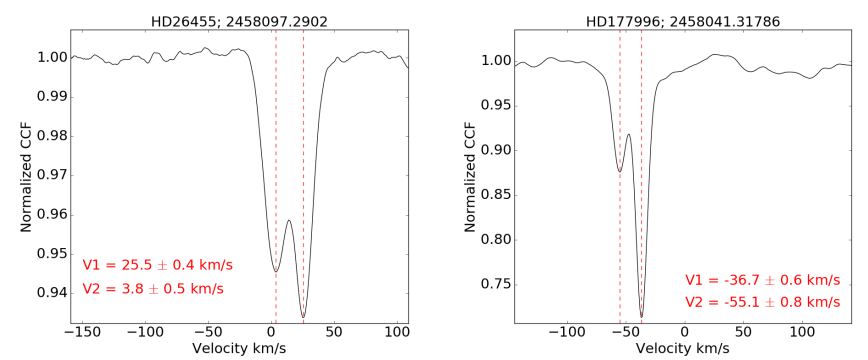

Fig. 6. Double-peaked CCF of two SALT-HRS spectra of HD 26455 (left) and HD 177996 (right).

The derived orbital period most likely corresponds to the inner orbit, since Tokovinin et al. (2006) and Tokovinin (2014), among others, have shown the absence of outer periods shorter than $\sim 1000$ days in triple systems with dwarfs of F-type or lower. This means that the WD companion is in an outer and longer orbit. According to the stability criterion discussed by Tokovinin (2014), the ratio between the long period and the short period must be higher than 4.7, so the outer orbit of HD 114520 must have a period of at least 2058 days, although it could be much longer.

The residuals of the fit do not show any long trend that can help us confirm the presence of a third body, but it should be noted that our data covers a period only slightly longer than the minimum stable outer period of this system. This contrasts with the more than 31 years of time coverage that we have for HD 48565, thanks to the CORAVEL and HERMES monitoring effort. Additionally, the residuals are in phase with the orbit, which could hide the long-period and low-amplitude signal of a third body. Since we are not able to detect the orbit of the WD companion, we do not include this object in the subsequent analysis.

\section{4. $H D 26455$ and HD 177996}

These two systems show a double-peaked CCF (see Fig. 6) in at least one of the available SALT spectra. The Ba star must be an SB2 like HD 114520, but we do not have enough data yet to solve for the different components. For both systems, there is no indication that the second component of the SB2 spectra is the expected WD companion. This suggests that both of these systems must be triple systems as well.

\subsection{Binaries with incomplete orbital phase coverage and unconfirmed binaries}

We could not determine orbits for 33 out of 60 objects in our sample. However, binary motion is clearly detected for 13 of them. Some of these were already mentioned as spectroscopic binaries in North et al. (2000), but we include them again in the present paper for the sake of completeness. To decide whether a star is member of a binary system or not, we use the following criterion: when the standard deviation of the observations, $\operatorname{std}(\mathrm{RV})$, is higher than three times the average error bar, $\sigma(\mathrm{RV})$, we consider the star to present variability. Table 2 summarises the results. The confirmed binaries are marked in boldface and their radial velocities are shown in Fig. B.26.

Additionally, Fig. B.27, shows the radial velocity data of the 20 objects for which we cannot prove binary motion yet. In some cases, this is due to the lack of data (e.g. HD 105671 in Fig. B.27). In others, the radial-velocity changes hint at binary motion, but the measured RV dispersion is still too small for a firm conclusion (e.g. HD 220117 in Fig. B.27).
Table 2. Spectroscopic binaries with no orbits yet available (flagged in bold face and as "SB" in the last column) and objects with no variability detected.

\begin{tabular}{|c|c|c|c|}
\hline Name & $\begin{array}{l}\operatorname{std}(\mathrm{RV}) \\
{\left[\mathrm{km} \mathrm{s}^{-1}\right]}\end{array}$ & $\begin{array}{l}3 \sigma(\mathrm{RV}) \\
{\left[\mathrm{km} \mathrm{s}^{-1}\right]}\end{array}$ & Comments \\
\hline BD-11 ${ }^{\circ} 3853$ & 2.37 & 0.37 & SB \\
\hline CD-62 ${ }^{\circ} 1346$ & 2.03 & 1.70 & SB \\
\hline HD $2454^{(1)}$ & 0.5863 & 0.1917 & SB \\
\hline HD 6434 & 0.44 & 0.95 & \\
\hline HD 9529 & 0.59 & 1.52 & \\
\hline HD $13555^{(2)}$ & 0.14 & 0.33 & \\
\hline HD 18853 & 2.43 & 1.03 & SB \\
\hline HD 26455 & 5.15 & 1.63 & SB2 \\
\hline HD 31732 & 0.30 & 1.23 & \\
\hline HD 35296 & 0.24 & 0.59 & \\
\hline HD 60532 & 0.15 & 0.31 & \\
\hline HD 69578 & 17.99 & 1.65 & SB \\
\hline HD 82328 & 0.13 & 0.25 & \\
\hline HD 92545 & 0.50 & 0.42 & SB \\
\hline HD 101581 & 0.10 & 0.88 & \\
\hline HD 103840 & 0.52 & 0.97 & \\
\hline HD 104342 & 0.58 & 1.17 & \\
\hline HD 105671 & 0.31 & 0.94 & \\
\hline HD 109490 & 14.67 & 7.54 & SB \\
\hline HD 113402 & 1.06 & 2.91 & \\
\hline HD 117288 & 0.50 & 1.08 & \\
\hline HD 124850 & 0.42 & 0.51 & \\
\hline HD 130255 & 0.21 & 0.43 & \\
\hline HD 146800 & 0.21 & 0.99 & \\
\hline HD 170149 & 0.42 & 1.47 & \\
\hline HD 177996 & 9.53 & 1.18 & SB2 \\
\hline HD 188985 & 1.03 & 0.69 & SB \\
\hline HD 202400 & 4.03 & 4.21 & \\
\hline HD 205156 & 1.40 & 1.18 & SB \\
\hline HD 219899 & 0.36 & 0.96 & \\
\hline HD 220117 & 0.17 & 0.47 & \\
\hline HD 222349 & 3.26 & 1.35 & SB \\
\hline HD 224621 & 6.32 & 0.98 & SB \\
\hline
\end{tabular}

Notes. The second column ( $\operatorname{std}(\mathrm{RV}))$ gives the standard deviation of the $\mathrm{RV}$ points and the third column $(3 \sigma(\mathrm{RV}))$ gives three times the average error bar considering all the RV points. We claim that a star is in a binary system when the number in the second column is higher than that in the third column. ${ }^{(1)} \operatorname{std}(\mathrm{RV})$ and $3 \sigma(\mathrm{RV})$ were computed using only HERMES data. Binarity is clear from this data set, while the CORAVEL RV points are very scattered (see third panel of Fig. B.26). ${ }^{(2)} \operatorname{std}(\mathrm{RV})$ and $3 \sigma(\mathrm{RV})$ were computed excluding the second CORAVEL RV point, which we considered an outlier (see third panel of Fig. B.27).

\section{Hertzsprung-Russell diagram}

We developed a method for obtaining the atmospheric parameters and luminosities of a sample of more than $400 \mathrm{Ba}$ stars by modelling their spectral energy distributions (SEDs; Escorza et al. 2017). SEDs are mainly sensitive to the effective temperature, and provide only a limited amount of information about other parameters, especially about metallicity. In this work, we derived the stellar parameters of our main-sequence and subgiant stars from HERMES high-resolution spectra whenever available. We used the вAcchus pipeline (Masseron et al. 2016), which uses Turbospectrum (Alvarez \& Plez 1998; Plez 2012), a 1D local thermodynamic equilibrium (LTE) spectral-synthesis code. An additional check based on the equilibrium of excitation 
Table 3. Effective temperature $\left(T_{\text {eff }}\right)$, surface gravity $(\log g)$, metallicity $([\mathrm{Fe} / \mathrm{H}])$, and microturbulence velocity $(\xi)$ of the objects with a determined orbit.

\begin{tabular}{|c|c|c|c|c|c|c|c|c|c|}
\hline Name & Type & $T_{\text {eff }}[\mathrm{K}]$ & $\log g[\mathrm{dex}]$ & {$[\mathrm{Fe} / \mathrm{H}][\mathrm{dex}]$} & $\xi\left[\mathrm{km} \mathrm{s}^{-1}\right]$ & Ref. & $E(B-V)$ & $L / L_{\odot}$ & $M / M_{\odot}$ \\
\hline BD- $10^{\circ} 4311$ & $\mathrm{sgCH}$ & $5858 \pm 50$ & $3.9 \pm 0.3$ & $-0.65 \pm 0.10$ & $1.25 \pm 0.07$ & This work & $0.042 \pm 0.015$ & $1.99 \pm 0.05$ & $0.80 \pm 0.10$ \\
\hline BD-18255 & $\mathrm{dBa}$ & $6800 \pm 400$ & $4.3 \pm 0.3$ & $-0.18 \pm 0.05$ & $2.4 \pm 0.5$ & L\&B91 & $0.015 \pm 0.005$ & $4.2 \pm 1.2$ & $1.30 \pm 0.15$ \\
\hline $\mathrm{BD}+18^{\circ} 5215$ & $\mathrm{dBa}$ & $6452 \pm 50$ & $4.4 \pm 0.4$ & $-0.50 \pm 0.09$ & $0.82 \pm 0.09$ & This work & $0.014 \pm 0.007$ & $1.86 \pm 0.11$ & $1.10 \pm 0.10$ \\
\hline HD 15306 & $\mathrm{dBa}$ & $6898 \pm 50$ & $3.8 \pm 0.2$ & $-0.40 \pm 0.14$ & $0.9 \pm 0.3$ & This work & $0.037 \pm 0.007$ & $8.7 \pm 2.3$ & $1.30 \pm 0.10$ \\
\hline HD 22589 & $\mathrm{gBa}$ & $5672 \pm 56$ & $3.9 \pm 0.2$ & $-0.07 \pm 0.09$ & $1.20 \pm 0.04$ & This work & $0.048 \pm 0.008$ & $6.9 \pm 0.8$ & $1.30 \pm 0.10$ \\
\hline HD 24864 & $\mathrm{dBa}$ & $6337 \pm 95$ & $3.82 \pm 0.18$ & $0.0 \pm 0.11$ & $1.0 \pm 1.0$ & $\mathrm{~K}+13$ & $0.0+0.005$ & $9.2 \pm 0.6$ & $1.38 \pm 0.06$ \\
\hline HD 34654 & $\mathrm{dBa}$ & $6174 \pm 50$ & $4.4 \pm 0.3$ & $-0.09 \pm 0.07$ & $1.36 \pm 0.04$ & This work & $0.012 \pm 0.008$ & $1.8 \pm 0.3$ & $1.19 \pm 0.05$ \\
\hline HD 48565 & $\mathrm{dBa}$ & $6030 \pm 100$ & $3.8 \pm 0.3$ & $-0.6 \pm 0.3$ & $1.3 \pm 0.3$ & K\&G14 & $0.018 \pm 0.010$ & $2.8 \pm 0.3$ & $0.92 \pm 0.05$ \\
\hline HD 50264 & $\mathrm{dBa}$ & $5800 \pm 100$ & $4.2 \pm 0.2$ & $-0.34 \pm 0.08$ & $1.0 \pm 0.3$ & P\&J03 & $0.002 \pm 0.010$ & $0.89 \pm 0.08$ & $0.90 \pm 0.10$ \\
\hline HD 76225 & $\mathrm{dBa}$ & $6340 \pm 50$ & $3.9 \pm 0.2$ & $-0.37 \pm 0.08$ & $1.30 \pm 0.06$ & This work & $0.053 \pm 0.010$ & $5.9 \pm 0.7$ & $1.21 \pm 0.06$ \\
\hline HD 87080 & $\mathrm{sgCH}$ & $5483 \pm 50$ & $3.6 \pm 0.3$ & $-0.60 \pm 0.08$ & $1.20 \pm 0.04$ & This work & $0.028 \pm 0.012$ & $12.5 \pm 0.8$ & $1.6 \pm 0.2$ \\
\hline HD 89948 & $\mathrm{sgCH}$ & $6000 \pm 400$ & $4.0 \pm 0.3$ & $-0.13 \pm 0.05$ & $1.8 \pm 0.5$ & L\&B91 & $0.0 \pm 0.003$ & $1.36 \pm 0.07$ & $0.95 \pm 0.08$ \\
\hline HD 95241 & $\mathrm{dBa}$ & $5837 \pm 50$ & $3.6 \pm 0.2$ & $-0.37 \pm 0.07$ & $1.36 \pm 0.04$ & This work & $0.0+0.008$ & $7.8 \pm 0.8$ & $1.30 \pm 0.10$ \\
\hline HD 98991 & $\mathrm{dBa}$ & $6388 \pm 50$ & $2.7 \pm 0.4$ & $-0.44 \pm 0.08$ & $0.07^{(1)}$ & This work & $0.0+0.014$ & $14.7 \pm 1.0$ & $1.45 \pm 0.08$ \\
\hline HD 106191 & $\mathrm{dBa}$ & $5946 \pm 50$ & $4.3 \pm 0.4$ & $-0.29 \pm 0.10$ & $1.21 \pm 0.06$ & This work & $0.010 \pm 0.010$ & $2.0 \pm 0.2$ & $0.95 \pm 0.05$ \\
\hline HD 107574 & $\mathrm{dBa}$ & $6340 \pm 100$ & $3.6 \pm 0.5$ & $-0.80 \pm 0.25$ & $1.8 \pm 0.3$ & $\mathrm{~N}+94$ & $0.0+0.005$ & $5.6 \pm 0.2$ & $1.11 \pm 0.05$ \\
\hline HD 120620 & $\mathrm{gBa}$ & $4831 \pm 50$ & $3.0 \pm 0.3$ & $-0.29 \pm 0.10$ & $1.11 \pm 0.05$ & This work & $0.055 \pm 0.013$ & $12.2 \pm 1.6$ & $1.2 \pm 0.2$ \\
\hline HD 123585 & $\mathrm{sgCH}$ & $6047 \pm 100$ & $3.5 \pm 0.5$ & $-0.50 \pm 0.25$ & $1.8 \pm 0.3$ & $\mathrm{~N}+94$ & $0.054 \pm 0.012$ & $3.5 \pm 1.0$ & $1.00 \pm 0.10$ \\
\hline HD 127392 & $\mathrm{sgCH}$ & $5600 \pm 300$ & $3.9 \pm 0.3$ & $-0.52 \pm 0.05$ & $2.6 \pm 0.5$ & L\&B91 & $0.011 \pm 0.011$ & $0.98 \pm 0.13$ & $0.8 \pm 0.3$ \\
\hline HD 141804 & $\mathrm{sgCH}$ & $6000 \pm 400$ & $3.5 \pm 0.3$ & $-0.41 \pm 0.05$ & $1.7 \pm 0.5$ & L\&B91 & $0.0+0.003$ & $1.77 \pm 0.07$ & $0.9 \pm 0.2$ \\
\hline HD 147609 & $\mathrm{dBa}$ & $6411 \pm 50$ & $3.9 \pm 0.5$ & $-0.23 \pm 0.09$ & $1.26 \pm 0.07$ & This work & $0.044 \pm 0.007$ & $10.2 \pm 1.3$ & $1.40 \pm 0.10$ \\
\hline HD 150862 & $\mathrm{sgCH}$ & $6182 \pm 50$ & $4.1 \pm 0.3$ & $-0.33 \pm 0.08$ & $0.94 \pm 0.06$ & This work & $0.006 \pm 0.006$ & $2.2 \pm 0.2$ & $1.04 \pm 0.05$ \\
\hline HD 182274 & $\mathrm{sgCH}$ & $6327 \pm 50$ & $4.3 \pm 0.2$ & $-0.32 \pm 0.09$ & $1.05 \pm 0.06$ & This work & $0.012 \pm 0.007$ & $2.06 \pm 0.14$ & $1.09 \pm 0.05$ \\
\hline HD 207585 & $\mathrm{sgCH}$ & $5400 \pm 300$ & $3.3 \pm 0.3$ & $-0.57 \pm 0.05$ & $1.8 \pm 0.5$ & L\&B91 & $0.0+0.003$ & $3.8 \pm 0.8$ & $0.96 \pm 0.15$ \\
\hline HD 216219 & $\mathrm{gBa}$ & $5900 \pm 50$ & $3.6 \pm 0.3$ & $-0.17 \pm 0.08$ & $1.42 \pm 0.04$ & This work & $0.042 \pm 0.009$ & $9.9 \pm 0.8$ & $1.45 \pm 0.10$ \\
\hline HD 221531 & $\mathrm{dBa}$ & $6460 \pm 100$ & $4.2 \pm 0.5$ & $-0.30 \pm 0.25$ & $2.4 \pm 0.3$ & $\mathrm{~N}+94$ & $0.0+0.004$ & $4.7 \pm 1.4$ & $1.20 \pm 0.10$ \\
\hline
\end{tabular}

Notes. Column 7 gives the reference of the adopted stellar parameters when no HERMES spectra were available. Column 8 gives the line-of-sight extinction, and Cols. 9 and 10 list the derived luminosities and masses. ${ }^{(1)}$ Uncertain value due to the low signal-to-noise ratio of the spectrum around $\mathrm{Fe}$ lines.

References. For the spectroscopic stellar parameters: L\&B91: Luck \& Bond (1991); K+13: Kordopatis et al. (2013); K\&G14: Karinkuzhi \& Goswami (2014); P\&J03: Pereira \& Junqueira (2003); N+94: North et al. (1994).

and ionisation iron abundances derived from $\mathrm{Fe}_{\mathrm{I}}$ and $\mathrm{Fe}$ II lines was performed to ensure the consistency of the derived atmospheric parameters (see Karinkuzhi et al. 2018 for more details). The Fe I and Fe II lines that we used are listed in Jorissen et al. (2019). The derived effective temperature $\left(T_{\text {eff }}\right)$, surface gravity $(\log g)$, metallicity $([\mathrm{Fe} / \mathrm{H}])$, and microturbulence velocity $(\xi)$ are presented in Table 3. In this manuscript metallicity is expressed with respect to the solar value, as given by Asplund et al. (2009). We did this analysis for the 25 stars with a determined SB1 orbit and for HD 48565 (since there is a signature of only one star in its spectra), but we did not include HD 114520. For targets with no HERMES spectra available, or when the signal-to-noise ratio of the available spectra was not sufficient to determine accurate stellar parameters, we used the spectroscopic parameters from the literature. The relevant references are also included in Table 3.

Once the stellar parameters were derived, we used the SEDfitting tool described in Escorza et al. (2017) to find the bestfitting MARCS model atmosphere (Gustafsson et al. 2008) to the photometry of each target and to obtain the total line-of-sight extinction $(E(B-V))$. We used the parameter ranges obtained from the spectroscopic analysis (see Table 3 ) to limit $T_{\text {eff }}$ and $\log g$, and we fixed the metallicity to the closest available in the MARCS grid $(0.0,-0.25,-0.5$, or -0.75$)$; instead, $E(B-V)$ was the only parameter kept completely free. We integrated the best-fitting SED models over all wavelengths and used distances from Bailer-Jones et al. (2018), based on Gaia DR2 parallaxes (Lindegren et al. 2018), to compute accurate luminosities. We note that the Gaia DR2 parallaxes were derived from a single- star astrometric solution and all our stars are binaries. However, our orbital periods are different from one year and we do not expect this to have a big impact. Table 3 includes the derived extinction and luminosities. The luminosity errors come from the distance errors and from the error of the integrated flux. To obtain the error of the integrated flux, we integrated 1000 additional well-fitting SED models and used the standard deviation of these new fluxes as $1 \sigma$ uncertainty on the flux. We chose these 1000 models by combining stellar parameters randomly chosen from normal distributions centred around the spectroscopic values and within the uncertainties.

Figure 7 shows the position on the HRD of all the dwarf and subgiant $\mathrm{Ba}$ stars (pink squares) and $\mathrm{CH}$ stars (blue triangles) for which we could constrain an orbit. In these plots, we keep the classification found in the literature for each star. However, as mentioned before, the distinction between $\mathrm{dBa}$ and sgCH stars is not very clear, and some targets have been classified as both in different studies (see Table A.1). In order to decide in which group to include a star with two classifications in the literature, we used the following criterion: when the star had $[\mathrm{Fe} / \mathrm{H}]$ lower that -0.5 , we classified it as a $\mathrm{CH}$ star, and when it was higher as a Ba star. This is a classical criterion, generally used for the giant counterparts since $\mathrm{CH}$ stars were defined as metal-deficient Ba stars (see e.g. Luck \& Bond 1991 or Vanture $1992 \mathrm{a}, \mathrm{b}, \mathrm{c})$ with $[\mathrm{Fe} / \mathrm{H}]$ values ranging from -0.50 to -1.7 (Wallerstein \& Knapp 1998). We note, however, that this classification based on metallicity is not fully satisfactory (e.g. Yamashita 1975), and should be revised in the future. For 

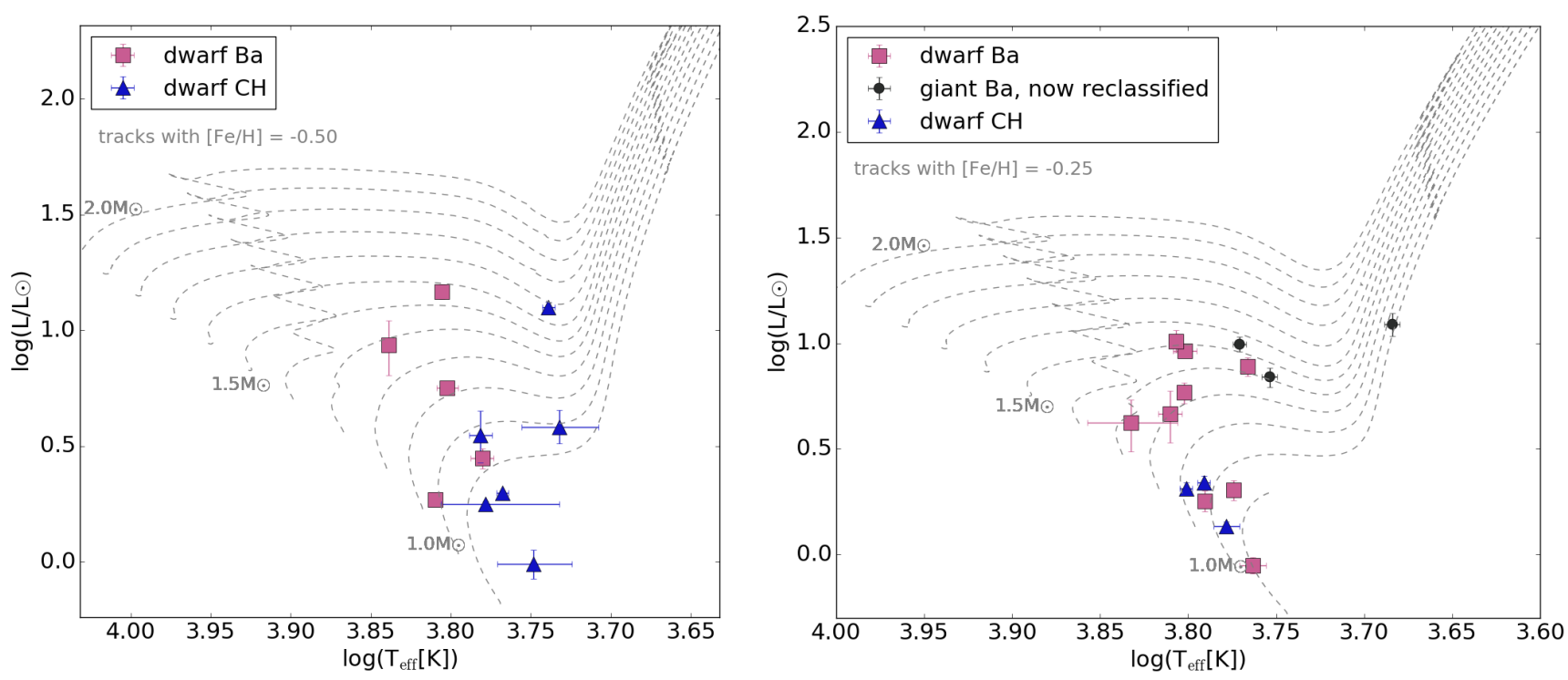

Fig. 7. Hertzsprung-Russell diagram of the dwarf and subgiant Ba (pink squares) and $\mathrm{CH}$ (blue triangles) stars for which orbital parameters were derived in this work. The three stars initially classified as giants are plotted with black circles. The objects are distributed in two HRDs to plot them with evolutionary tracks of the most appropriate metallicity: $[\mathrm{Fe} / \mathrm{H}]=-0.50($ left $)$ and $[\mathrm{Fe} / \mathrm{H}]=-0.25($ right $)$. The evolutionary tracks were computed with the STAREVOL code (Siess et al. 2000; Siess \& Arnould 2008).

example, $\mathrm{HD} 89948$ with $[\mathrm{Fe} / \mathrm{H}]=-0.13$ is referred to as a $\mathrm{CH}$ star in the literature, while $\mathrm{HD} 48565$ with $[\mathrm{Fe} / \mathrm{H}]=-0.6$ is defined as a Ba star.

Following this criterion, HD 87080 appears now among the main-sequence $\mathrm{CH}$ stars, while BD-18 255 and HD 50264 appear among the Ba dwarfs. If only one classification was found in the literature, we kept it regardless of the metallicity value. The three stars initially classified as gBa (HD 22589, HD 120620, and HD 216219) in the catalogue by Lü et al. (1983) are represented in Fig. 7 with black circles. Their position in the HRD is more compatible with subgiants as they have not ascended the RGB yet. From now on, we include these three stars in our dwarf and subgiant $\mathrm{Ba}$ star sample. Figure 7 is divided into two panels. The left panel shows a HRD with all the stars in our sample with $[\mathrm{Fe} / \mathrm{H}] \lesssim$ -0.40 and STAREVOL (Siess et al. 2000; Siess \& Arnould 2008) evolutionary tracks computed with $[\mathrm{Fe} / \mathrm{H}]=-0.50$. The HRD in the right panel shows stars with $[\mathrm{Fe} / \mathrm{H}] \gtrsim-0.40$ and STAREVOL evolutionary tracks computed with $[\mathrm{Fe} / \mathrm{H}]=-0.25$.

A noticeable feature of our HR diagrams is the location of some of our most massive objects with respect to the Hertzsprung gap. A considerable fraction of our objects appear in regions of the HRD where evolution is fast and not many stars are expected to be observed. To illustrate this more clearly, in Fig. 8 we have coloured our evolutionary tracks of $[\mathrm{Fe} / \mathrm{H}]=-0.25$ according to their apparent evolutionary speed. We computed this speed as the distance between two points on a track divided by the age difference between them. Stellar evolution on the dark blue parts of a track is much slower than on the light green parts. Although this is a simple approach, it is able to show that several dBa stars occupy rapid phases of evolution.

Finally, to obtain individual masses for our primary stars, we used the same method as in Escorza et al. (2017). We derived the mass by comparing the position of the stars on the HRD with a grid of evolutionary models computed with the STAREVOL code (Siess et al. 2000; Siess \& Arnould 2008). The grid covers a wide range of masses, from $0.9 M_{\odot}$ to $4.0 M_{\odot}$ with a mass step of $0.1 M_{\odot}$, and four different metallicities, $[\mathrm{Fe} / \mathrm{H}]=0.0$, $-0.25,-0.5$ and -1.0 . To compute these evolutionary models, we included a mass-dependent formulation for the overshooting on

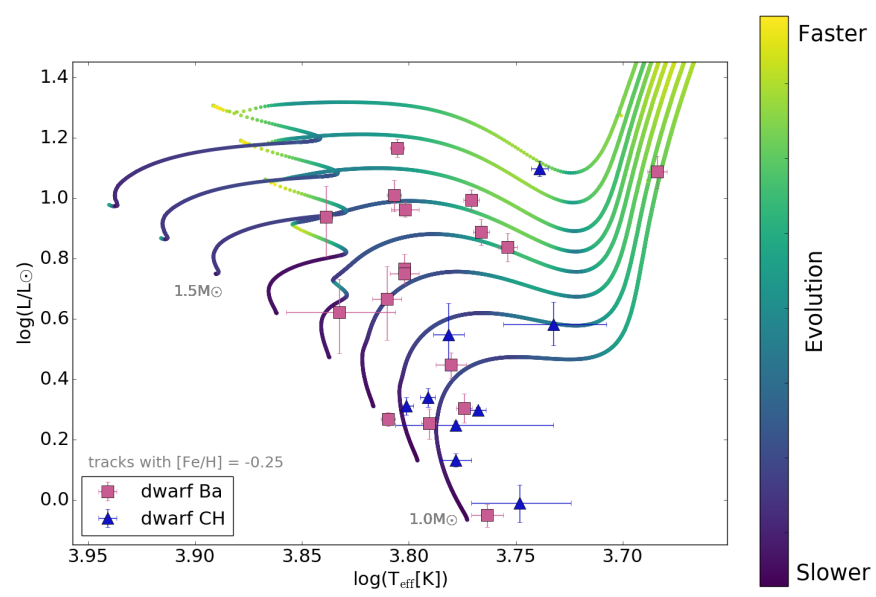

Fig. 8. Same as the right panel of Fig. 7, but with stars of all metallicities included. The colour of the tracks is proportional to an evolutionary velocity computed as the distance between two points divided by the age difference between them. Dark blue colours trace much slower evolution than light green ones.

top of the convective core, following Claret \& Torres (2018), and considered a wind prescription as described by Schröder \& Cuntz (2007) until the beginning of the AGB phase. We also included some overshooting at the base of the convective envelope, following the exponential decay expression of Herwig et al. (1997) with $f_{\text {over }}=0.1$. Finally, we used a grey atmosphere surface boundary condition. The derived masses are presented in Table 3 . The uncertainty on the mass is obtained by deriving the upper and lower mass limits given the error bars of the spectroscopic parameters.

\section{Discussion}

\subsection{Eccentricity-period diagram}

Figure 9 presents the eccentricity-period $(e-\log P)$ diagram of $\mathrm{Ba}$ stars at different evolutionary stages and metallicities. The pink squares are the stars flagged as Ba dwarfs and subgiants, 

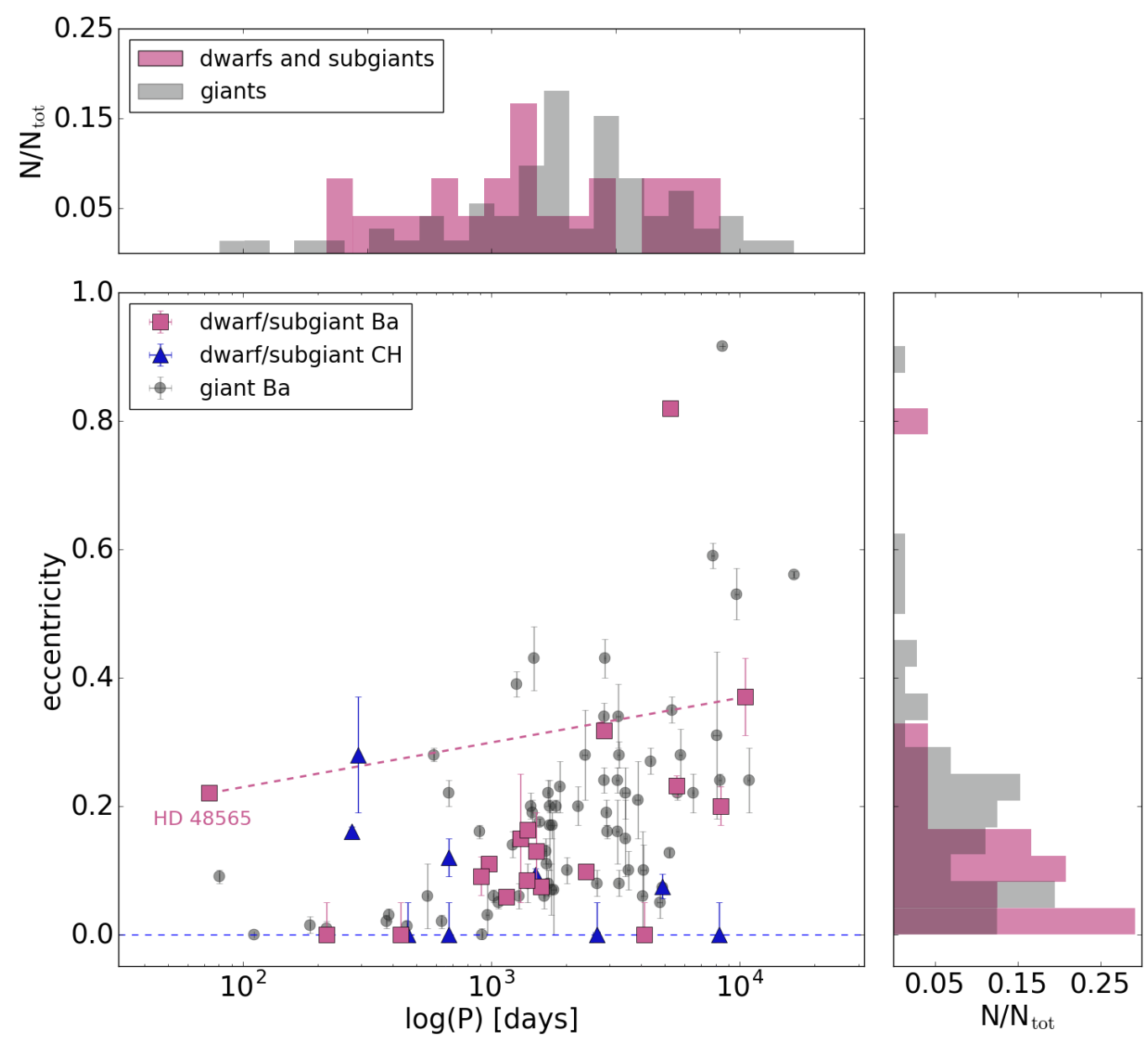

Fig. 9. Eccentricity-period diagram of dwarf and subgiant Ba stars (pink squares), including HD 22589, HD 120620, and HD 216219, initially called giants, and of dwarf and subgiant $\mathrm{CH}$ stars (blue triangles). The two orbits of the triple system HD 48565 are linked with a dashed line. Orbits of giant $\mathrm{Ba}$ are included as grey circles for comparison (Jorissen et al. 2019). Top and right panels: normalised histograms of log $P$ and $e$, respectively.

including HD 22589, HD 120620, and HD 216219, and the blue triangles are the dwarf and subgiant $\mathrm{CH}$ stars. The two orbits of the triple system HD 48565 are linked with a dashed line since we cannot be sure which orbit hosts the WD. The grey circles and crosses respectively represent $\mathrm{Ba}$ and $\mathrm{CH}$ giant orbits from the literature (Jorissen et al. 2016, 2019), which we include for comparison. The smaller panels in Fig. 9, top and right, show histograms of the period and eccentricity distributions, respectively. In these diagrams the giant $\mathrm{Ba}$ and $\mathrm{CH}$ stars are represented in grey and the dwarf and the subgiant $\mathrm{Ba}$ and $\mathrm{CH}$ stars are represented together in pink. Figure 9 shows the following:

- The ranges of observed periods and eccentricities are similar for all type of objects.

- At short periods $(P<1000 \mathrm{~d})$, we observe circular orbits and a few remarkably eccentric ones. Among the new orbits, two $\mathrm{CH}$ dwarfs stand out: HD $87080(P=274.3 \pm 1.9 \mathrm{~d}, e=$ $0.161 \pm 0.007)$ and HD $150862(P=291 \pm 4 \mathrm{~d}, e=0.28 \pm$ $0.09)$. We do not have many radial-velocity points for these objects, but the eccentric orbital model clearly fits better, and the HERMES point confirms the CORAVEL eccentric orbit in both cases (see Figs. B.10 and B.21). The inner orbit of HD 48565 stands out as well among the short period binaries. This is likely a result of dynamical interaction in the triple system (see discussion in Sect. 4.2).

- At longer periods $(P>1000 \mathrm{~d})$, all barium giants seem to be eccentric, but among the new systems we find three with zero eccentricity.
- Finally, we find giant systems with very long periods $(P>10000 \mathrm{~d})$ and large eccentricities. Only one Ba dwarf (HD 95241) populates that region of the diagram, but among our "uncovered" orbits, there are some confirmed binaries that will have very long periods as well.

Overall, the $e-\log P$ diagram of the dwarf and subgiant stars does not show specific features to distinguish them from their giant counterparts, apart from the presence of a few largeeccentricity systems at short periods among the $\mathrm{CH}$ dwarfs.

\section{2. $\mathrm{dBa}$ vs. $\mathrm{sgCH}$}

Figure 7 corroborates the finding of our earlier work (Escorza et al. 2017): there is no evolutionary distinction between dwarf $\mathrm{Ba}$ stars and subgiant $\mathrm{CH}$ stars. Some $\mathrm{dBa}$ stars are not real dwarfs and many $\operatorname{sgCH}$ stars are located on the main sequence and are not subgiants. Additionally, we do not see a compelling metallicity difference between the two classes of stars. The stars classified in the literature as dwarf Ba stars have, on average, a higher metallicity than those classified as subgiant $\mathrm{CH}$ stars, but we find outliers in both subsamples.

These two classes of stars look very similar in many aspects, but there is a noticeable difference in their location on the HRD. The dwarf $\mathrm{CH}$ stars are located on the bottom right part of the main sequence, so they have a lower temperature and are less massive than the dwarf $\mathrm{Ba}$ stars. $\mathrm{CH}$ stars are characterised by strong $\mathrm{G}$ bands due to the $\mathrm{CH}$ molecule 


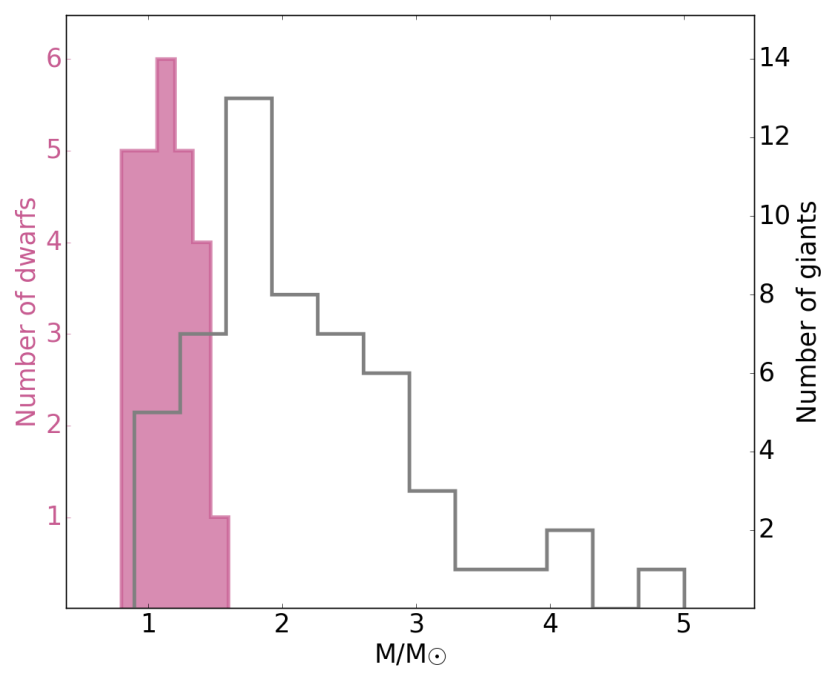

Fig. 10. Mass distribution of dwarf and subgiant $\mathrm{Ba}$ and $\mathrm{CH}$ stars (solid pink) and of Ba giants (grey) from Jorissen et al. (2019).

(Keenan 1942) that might not be detectable in the spectra of hotter main-sequence stars. This might be causing a bias in the classification of s-process-enhanced main-sequence stars making $\mathrm{CH}$ dwarfs appear as the low-mass analogues of Ba dwarfs. These families of stars would benefit from a new and systematic spectroscopic classification, but this is beyond the scope of this study. We do not see this effect on the giant counterparts, since $\mathrm{Ba}$ and $\mathrm{CH}$ giants occupy the same region on the HRD (see Fig. 7 of Escorza et al. 2017).

\subsection{Mass distribution}

Figure 10 presents the mass distribution of $\mathrm{Ba}$ and $\mathrm{CH}$ stars before (pink histogram) and after (black histogram) they ascend the RGB. Only objects with determined orbital solutions are included in Fig. 10 (the same systems as in Fig. 9). The masses of the giants come from Jorissen et al. (2019). The mass distribution of Ba dwarf and subgiant stars peaks at a significantly lower value than the mass distribution of $\mathrm{Ba}$ giants, which peaks around $2 M_{\odot}$. Hence, the $\mathrm{Ba}$ and $\mathrm{CH}$ stars that we discuss in this paper will not evolve into the prototypical $\mathrm{Ba}$ giants that are observed, only into the least massive ones. The reason for this is likely an observational bias against hotter dwarf Ba stars. A-type stars are typically fast rotators, hence the lines appear broadened in their spectra, which makes detecting and quantifying the s-process overabundances difficult. Moreover, in this region of the main sequence, a wide variety of stellar processes can cause stellar variability (e.g. $\delta$ Scuti, $\gamma$ Doradus, and roAp stars) and chemical inhomogeneities (e.g. Am and Ap stars). These effects could wash out any signature of past mass-transfer in the spectra. It is likely that some of the $\delta$ Scuti stars with suspected WD companions studied by Murphy et al. (2018) gained enriched material from a former AGB companion, but a systematic highresolution spectral survey at short wavelength is needed to confirm this hypothesis.

\subsection{Mass function and companion masses}

The mass function relates the masses of the two components in a binary $\left(m_{1}\right.$ being the mass of the Ba star and $m_{2}$ being the mass of the WD companion in our case) and the inclination of the system $(\sin i)$ with the parameters derived from the spectroscopic orbit
Table 4. Parallaxes and orbital inclinations from the combination of spectroscopic parameters and HIPPARcos astrometric data and companion masses of the $\mathrm{Ba}$ and $\mathrm{CH}$ stars that passed the tests presented in Pourbaix \& Arenou (2001) and Jancart et al. (2005).

\begin{tabular}{lccc}
\hline \hline ID & $\varpi[\mathrm{mas}]$ & Inclination $\left[{ }^{\circ}\right]$ & Companion mass $\left[M_{\odot}\right]$ \\
\hline HD 34654 & $21.5 \pm 1.0$ & $80 \pm 4$ & $0.621 \pm 0.018$ \\
HD 50264 & $14.1 \pm 1.1$ & $109 \pm 5$ & $0.60 \pm 0.05$ \\
HD 89948 & $23.9 \pm 0.8$ & $102 \pm 3$ & $0.54 \pm 0.03$ \\
HD 123585 & $9.5 \pm 1.7$ & $64 \pm 13$ & $0.66 \pm 0.11$ \\
\hline
\end{tabular}

(period, $P$; eccentricity, $e$; and semi-amplitude, $K_{1}$ ) as follows:

$f(m)=\frac{m_{2}^{3}}{\left(m_{1}+m_{2}\right)^{2}} \sin ^{3} i=1.0361 \times 10^{-7} \cdot\left(1-e^{2}\right)^{3 / 2} K_{1}^{3} P\left[M_{\odot}\right]$,

with $P$ expressed in days and $K_{1}$ in $\mathrm{km} \mathrm{s}^{-1}$.

We derived the mass functions of our binaries in Sect. 3 (Col. 9 of Table 1) and the masses of the primary stars in Sect. 5 (Col. 9 of Table 3). We are only missing information about the orbital inclination to derive the mass of the WD companions. Following the methodology used in Pourbaix \& Jorissen (2000), Pourbaix \& Boffin (2003), and Jancart et al. (2005), among others, we combined our new orbital solutions with HIPPARcos astrometric data to derive the astrometric orbit of our spectroscopic binaries. This had been done for some of our targets before (Pourbaix \& Jorissen 2000); however, the statistical tests introduced later by Pourbaix \& Arenou (2001) and Jancart et al. (2005) were not applied at the time. We repeated the reprocessing of the HipPARcos astrometric data, and only four of our Ba and $\mathrm{CH}$ stars passed the statistical tests. The obtained parallaxes, inclinations and companion masses are presented in Table 4. Since the orbital periods of these targets are far from one year, we do not expect disagreement in the parallaxes with respect to the single-star solution. The obtained parallaxes are in good agreement with the Hipparcos (Perryman et al. 1997) and Gaia DR2 values (Lindegren et al. 2018). Additionally, the derived WD masses are compatible with current estimates for field WD masses (Kleinman et al. 2013) and with the mass distribution of WD companion of Ba giants obtained by Jorissen et al. (2019).

Only after the third data release of the Gaia satellite, which will include binary astrometric solutions, will we be able to obtain companion masses for our other systems. In the meantime, several assumptions can be made about the unknown parameters in order to model the observed cumulative distribution of the mass functions. The mass distribution of the primary stars $\left(\mathrm{m}_{1}\right)$ is described in our model as a Gaussian centred at a mass $\mu_{1}$ and with standard deviation $\sigma_{1}$. We allow these two parameters to vary within ranges compatible with Fig. 10. Additionally, it is safe to assume that the secondary stars are WDs, so their mass distribution is also described with a Gaussian characterised by $\mu_{2}$ and $\sigma_{2}$, which are also free parameters within ranges compatible with WD masses from Kleinman et al. (2013). Finally, we need a distribution of orbital inclinations. If we assume that orbital planes are randomly oriented in space and take into account projection effects, we can use the distribution

$i=\arccos (z)$,

where $z$ is the length of the projection of the unit normal vector along the line of sight and is uniformly distributed in the range $[-1,1]$. 


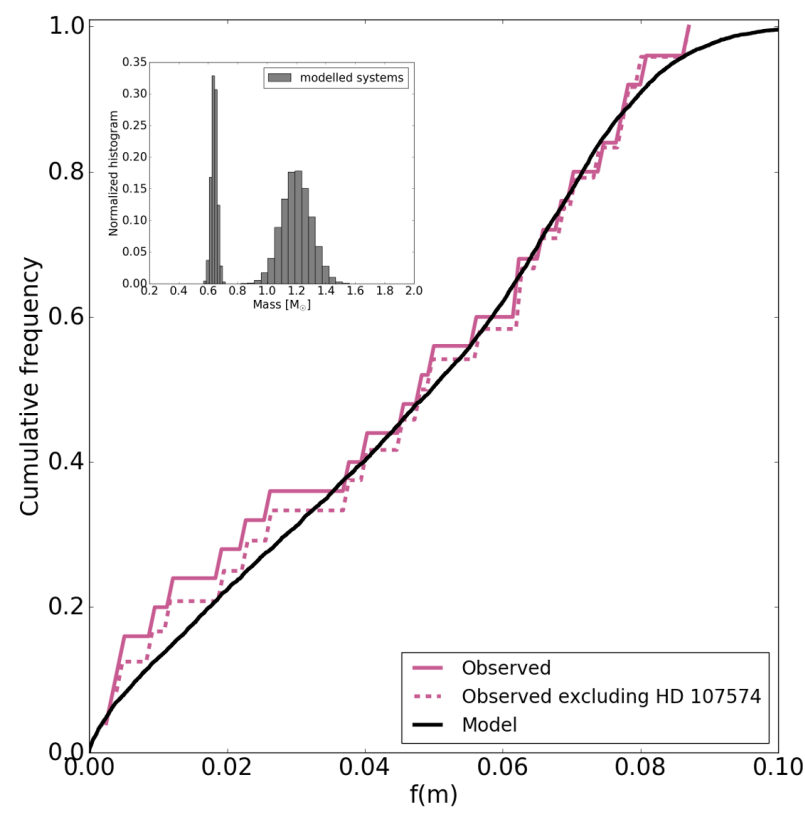

Fig. 11. Cumulative distribution of the mass functions of $\mathrm{Ba}$ and $\mathrm{CH}$ dwarf and subgiant stars (continuous pink line) and best fit to the distribution (dashed pink line). Giant Ba stars are shown in grey for comparison.

We generated 10000 random orbits and compared the modelled cumulative distribution with our observations to constrain the parameters that describe the mass distribution of the two stars in these systems. Figure 11 shows the comparison of the observed distribution (solid pink line) and the best-fitting model (solid black line). This has been found for a primary mass distribution centred at $1.2 M_{\odot}$ with $\sigma_{1}=0.1 M_{\odot}$ and a secondary (WD) mass distribution centred at $0.64 M_{\odot}$ with $\sigma_{2}=0.02 M_{\odot}$. The distributions are shown in the inset in the top left corner of Fig. 11. There is a small excess of low mass-functions with respect to the best-fitting model, which seems to be mostly caused by HD 107574, a target with a remarkably low massfunction compared with the others (see Table 3). The dashed pink line in Fig. 11 shows the observed cumulative distribution of mass functions when we exclude this object, and it seems to agree better with our best-fitting model. However, we do not have a reason to exclude it from our sample. Assuming our value for $m_{1}$ is correct, the low mass-function could be due to a very low companion mass, or to a very low orbital inclination (of about $16^{\circ}$ to host a $0.6 M_{\odot}$ WD), but we cannot distinguish between these two possibilities with the available information.

Webbink (1986) already suggested that the mass-function distribution of Ba stars can be fitted by a sample of orbits with a very narrow distribution of $Q$, where $f(m)=Q \sin ^{3} i$, and several studies have confirmed this for Ba giants (e.g. Jorissen et al. 1998, 2019). Our model (black solid line in Fig. 11) corresponds to a Gaussian distribution of $Q$ with $\mu_{Q}=0.077 M_{\odot}$ and $\sigma_{Q}=0.01 M_{\odot}$. The small sigma of the $Q$ distribution suggests that $m_{1}$ and $m_{2}$ could be correlated.

Finally, $Q$ can also be expressed as

$Q=m_{1} \frac{q^{3}}{(1+q)^{2}}\left[M_{\odot}\right]$

where $q=m_{2} / m_{1}$ is the mass ratio of the two stars. Applying the Lucy-Richardson inversion method (e.g. Boffin et al. 1992; Cerf \& Boffin 1994) to the distribution of $Q$ values, we can also obtain the distribution of $q$ (Fig. 12).

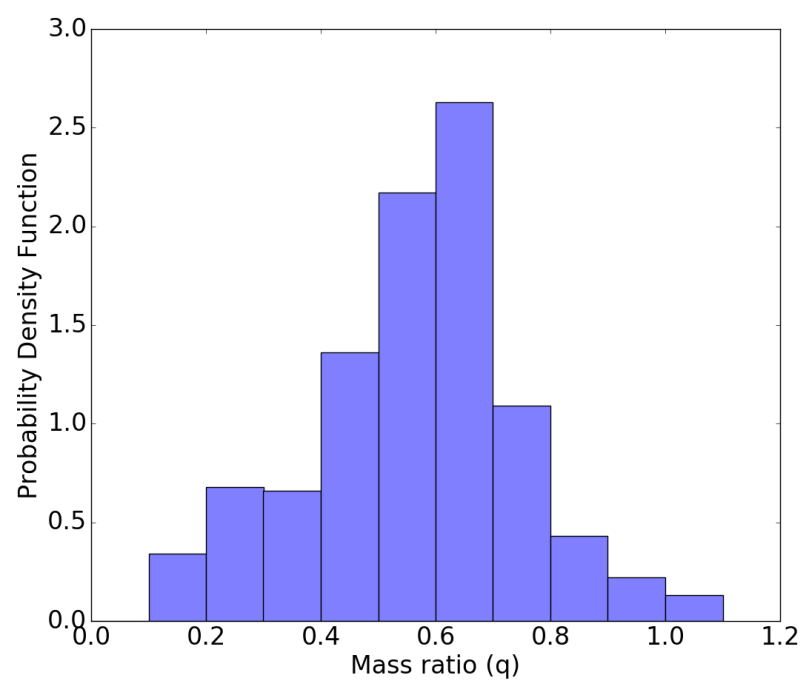

Fig. 12. Probability density function (PDF) of mass ratios of our sample of $\mathrm{Ba}$ and $\mathrm{CH}$ dwarf and subgiant stars.

\subsection{Comparison with evolutionary models}

Since the first $e-\log P$ diagrams of Ba giants were obtained, a great deal of research has been devoted to understanding them. The interaction between the former AGB star and its less evolved companion shapes the orbits in a way that cannot be closely reproduced by evolutionary models (e.g. Pols et al. 2003; Bonačić Marinović et al. 2008). However, Ba stars might have gone through a second stage of binary interaction when the dwarf ascended the RGB, which can also affect the eccentricity and the period of Ba giants.

In order to explore the evolutionary link between our Ba dwarfs and the observed low-mass Ba giants, we computed a grid of standard binary evolution models with the BINSTAR code (Siess et al. 2013). We used input parameters inspired by our sample of main-sequence $\mathrm{Ba}$ and $\mathrm{CH}$ stars. The systems that we modelled were formed by a primary main-sequence star with $M=1.5 M_{\odot}$ and metallicity $[\mathrm{Fe} / \mathrm{H}]=-0.25$, and a cool WD companion of $M=0.6 M_{\odot}$. We used seven initial orbital periods (100, 300, 600, 1000, 2000, 3000, and 10000 days) and four eccentricities $(0.2,0.4,0.6$, and 0.8$)$. We only followed the evolution of the primary star, for which we used a mass-loss prescription as described by Schröder \& Cuntz (2007) and a grey atmosphere as surface boundary condition. We considered standard binary interactions assuming that the stars are in solid rotation and that angular momentum evolution is governed by the effect of tides and mass loss. We considered that the wind carries away the specific orbital angular momentum of the star as in the Jeans mode. In Escorza et al. (2017) we showed that Ba giants accumulate in the He-clump, so we allowed the primary star to evolve until the onset of core He-burning and compared the results with known orbits of giants.

Figure 13 shows the final orbits of the models that reached the core He-burning phase (blue circles) together with observations of $\mathrm{Ba}$ giants (orange crosses) that have $M=1.5 M_{\odot}$ or lower. The grey triangles are the initial orbital parameters of the models. When the primary reaches the core He-burning phase, the initial and final orbital parameters are linked by a dashed line that shows the evolution of the orbit. Triangles that are not connected to an evolutionary track correspond to models in which the primary star leaves the RGB after losing most of its mass via Roche-lobe overflow (RLOF) and does not become one of the 


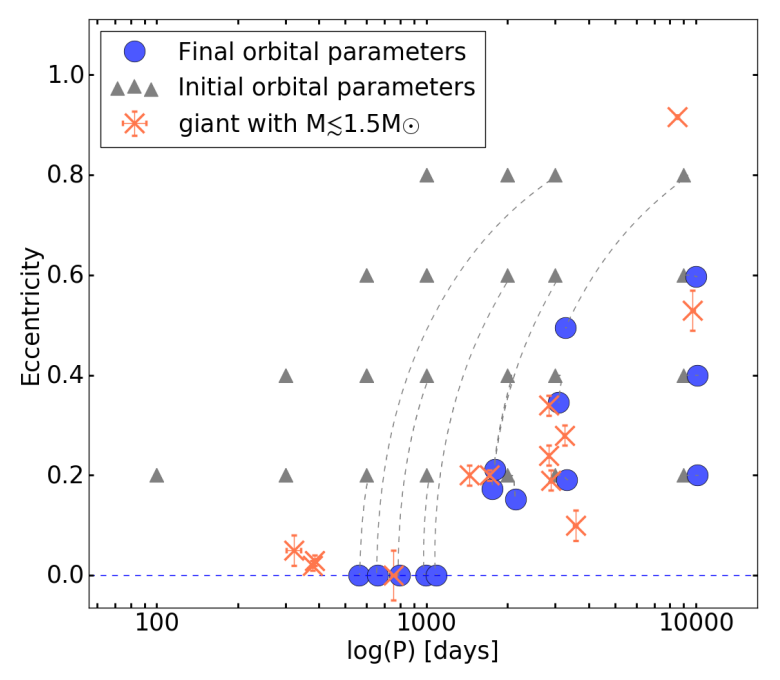

Fig. 13. Observed and modelled orbits of Ba giants after the binary interaction along the RGB phase. The triangles indicate the initial period an eccentricity of each BINSTAR model and the blue dots indicate the final orbital parameters at the time of core-He burning onset. The evolution of the models that reached the core-He burning phase is represented by a dashed grey line. Orange crosses are observed orbits of low-mass $\left(\lesssim 1.5 M_{\odot}\right)$ Ba and $\mathrm{CH}$ giants from Jorissen et al. (2019).

giant $\mathrm{Ba}$ systems that we observe. These models do not have a corresponding final orbit (blue circle) in the figure.

When we compare the observations with the model predictions for this primary mass, we find a few outlying objects. Three giant systems have periods shorter than those allowed by standard binary models of this mass $(P<700 \mathrm{~d})$. There is also one $\mathrm{Ba}$ giant with a very long period and a very high eccentricity that our models cannot reproduce. As the model with $P_{\text {init }}=10000$ days and $e_{\text {init }}=0.8$ shows, it is very difficult to keep such a high eccentricity after a stage of tidal interaction in the RGB. Another object mentioned in Jorissen et al. (2019), HD 134698, does not have a well-constrained orbit yet, but will probably occupy the same region of the $e-\log P$ diagram (the preliminary estimated period and eccentricity are 10005 days and 0.95). Binary interaction mechanisms, which we do not include in our standard binary calculations, need to be considered to reproduce all the observed $\mathrm{Ba}$ giant orbits.

\section{Summary and conclusions}

We combined radial velocity data from four different instruments to analyse a sample of 60 objects classified in the literature as dwarf $\mathrm{Ba}$ or subgiant $\mathrm{CH}$ stars. To our knowledge, this is the largest systematic radial-velocity survey of dwarf and subgiant $\mathrm{Ba}$ and related stars. We determined accurate orbital parameters for 27 binaries. Twenty-five of them are SB1s with a polluted primary and a suspected WD companion. Additionally, HD 48565 is a triple system, for which we constrained both the inner and outer orbit, and HD 114520 is an SB2. The latter must be a triple system as well since the two stars that contribute to the spectra are main-sequence stars enhanced in s-process elements (Gray et al. 2011). We determined the orbital parameters of the SB2 and concluded that the WD must be in an outer orbit of at least 2058 days, but we did not detect its signature in the residuals of the orbital fit as we did for HD 48565. In addition, we found two more SB2s, HD 26455 and HD 177996, but we could not constrain their orbits due to a lack of data. Among the remaining objects in the sample, we confirmed radial velocity variability of
11 of them, but again we did not have enough data to determine their orbital parameters. The monitoring of these long-period binaries will continue. The other 20 objects do not present clear variability according to the criterion adopted in Sect. 4.5.

We determined stellar parameters from HERMES highquality spectra or adopted literature values coming from highresolution spectroscopy to locate the primary stars of our SB1s on the HRD. We used distances derived by Bailer-Jones et al. (2018) from Gaia DR2 data to obtain accurate luminosities. From the comparison of the location of these stars on the HRD with STAREVOL evolutionary tracks, we have also derived their masses. Our spectroscopic metallicities show no clear distinction between stars classified in the literature as $\mathrm{dBa}$ or as $\mathrm{sgCH}$ stars and our HRD does not agree with the difference in evolutionary stage that their names suggest. However, we observe that main-sequence $\mathrm{CH}$ stars are cooler and less massive than main-sequence Ba stars. The HRD also shows that several targets occupy regions of fast stellar evolution where it is difficult to observe many stars.

When we compare our sample with a sample of Ba giants, we see that the mass distribution of main-sequence and subgiants stars peaks at much lower mass than the distribution of masses of their more evolved analogues. This means that the sample of dwarf and subgiant stars that we studied does not represent the precursor sample of the observed Ba giants. We associate this with an observational bias against the detection of hotter postmass-transfer main-sequence stars.

From our orbital analysis, we concluded that the periods and eccentricities of $\mathrm{Ba}$ and $\mathrm{CH}$ dwarfs lie on the same region of the $e-\log P$ diagram as the orbital elements of $\mathrm{Ba}$ giants. They also present high eccentricities at short periods like many other families of post-interaction binaries. Another product of the orbital analysis is the mass function of the systems. We combined our spectroscopic orbital elements with HipPARcos astrometric data and could obtain the orbital inclination of four of our systems. Using the mass functions, the primary masses that we determined and these inclinations, we derived the absolute mass of the four WD companions. The obtained values are consistent with field WD masses. The combination of spectroscopic orbital elements and astrometric data is very powerful, and with Gaia DR3 this method will allow us to put more stringent observational constraints to the formation and evolution of Ba stars.

Since we cannot determine absolute WD masses for all the systems yet, we also modelled the mass function distribution of our spectroscopic binaries, concluding that all these systems can be represented by a population of binary systems formed by primary main-sequence stars and WDs with very narrow and Gaussian mass distributions and orbital planes randomly oriented on the sky. The mass distribution obtained for the primaries peaked at $1.2 M_{\odot}$, which is in good agreement with the masses that we obtained from independent observations. The secondary mass distribution is an expected distribution of WD masses as well.

Finally, we used the BINSTAR binary evolution code to study the evolution of the orbital elements of our main-sequence stars along the RGB until they become the low-mass Ba giants that Jorissen et al. (2019) observed. Our models can explain the majority of the giant orbits with primary mass $\lesssim 1.5 M_{\odot}$, but we find four unexplained systems, three with short periods and modest eccentricities and one with a long period and a very high eccentricity. This might indicate that additional binary interaction mechanisms are also needed to explain the orbital evolution of low-mass Ba star systems along the RGB, independently of the previous interaction during the AGB phase of the now WD companion. 
Acknowledgements. This research has been funded by the Fonds voor Wetenschappelijk Onderzoek Vlaanderen (FWO) under contract ZKD1501-00-W01 and by the Belgian Science Policy Office under contract $\mathrm{BR} / 143 / \mathrm{A} 2 / \mathrm{STARLAB}$. A.E. is grateful to the staff members of the IvS (KU Leuven) and IAA (ULB) for the enriching discussions and valuable feedback and to all observers of the HERMES consortium for their time dedicated to the Mercator-HERMES long-term monitoring programme, and also to Dr. Silvia Toonen for the discussions about triple systems. D.K. acknowledges the financial support from SERB-DST, through the file number PDF/2017/002338. L.S. and D.P are Senior FNRS Research Associates. C.J. acknowledges funding from the European Research Council (ERC) under the European Union's Horizon 2020 research and innovation programme (grant agreement $\mathrm{N}^{\circ}$ 670519: MAMSIE). B.M. acknowledges support from the National Research Foundation (NRF) of South Africa. J.M. acknowledges the funding from the National Science Centre, Poland, through grant OPUS 2017/27/B/ST9/01940. This work has made use of data from the European Space Agency (ESA) mission Gaia (https://www. cosmos.esa.int/gaia) processed by the Gaia Data Processing and Analysis Consortium (DPAC, https://www.cosmos.esa.int/web/gaia/dpac/consortium). Funding for the DPAC has been provided by national institutions, in particular the institutions participating in the Gaia Multilateral Agreement. Some of the observations reported in this paper were obtained with the Southern African Large Telescope (SALT). Polish participation in SALT is funded by grant No. MNiSW DIR/WK/2016/07. This research has made use of the SIMBAD database, operated at CDS, Strasbourg, France.

\section{References}

Abate, C., Pols, O. R., \& Stancliffe, R. J. 2018, A\&A, 620, A63

Alvarez, R., \& Plez, B. 1998, A\&A, 330, 1109

Asplund, M., Grevesse, N., Sauval, A. J., \& Scott, P. 2009, ARA\&A, 47, 481

Bailer-Jones, C. A. L., Rybizki, J., Fouesneau, M., Mantelet, G., \& Andrae, R 2018, ApJ, 156, 58

Ballester, P. 1992, in European Southern Observatory Conference and Workshop Proceedings, eds. P. J. Grosb $\varnothing 1, \&$ R. C. E. de Ruijsscher, 41, 177

Baranne, A., Mayor, M., \& Poncet, J. L. 1979, Vistas Astron., 23, 279

Bidelman, W. P., \& Keenan, P. C. 1951, ApJ, 114, 473

Boffin, H. M. J., Paulus, G., \& Cerf, N. 1992, in Binaries as Tracers of Star Formation, eds. A. Duquennoy, \& M. Mayor, 26

Böhm-Vitense, E., Nemec, J., \& Proffitt, C. 1984, ApJ, 278, 726

Böhm-Vitense, E., Carpenter, K., Robinson, R., Ake, T., \& Brown, J. 2000, ApJ, 533, 969

Bonačić Marinović, A. A., Glebbeek, E., \& Pols, O. R. 2008, A\&A, 480, 797

Bond, H. E. 1974, ApJ, 194, 95

Bramall, D. G., Sharples, R., Tyas, L., et al. 2010, Proc. SPIE, 7735, 77354F

Bramall, D. G., Schmoll, J., Tyas, L. M. G., et al. 2012, Proc. SPIE, 8446, 84460A

Buckley, D. A. H., Swart, G. P., \& Meiring, J. G. 2006, Proc. SPIE, 6267, 62670Z Cerf, N., \& Boffin, H. M. J. 1994, Inverse Problems, 10, 533

Claret, A., \& Torres, G. 2018, ApJ, 859, 100

Crause, L. A., Sharples, R. M., Bramall, D. G., et al. 2014, Proc. SPIE, 9147, 91476T

Crawford, S. M., Still, M., Schellart, P., et al. 2010, Proc. SPIE, 7737, 773725

Duquennoy, A., Mayor, M., \& Halbwachs, J.-L. 1991, A\&AS, 88, 281

Edvardsson, B., Andersen, J., Gustafsson, B., et al. 1993, A\&A, 275, 101

Escorza, A., Boffin, H. M. J., Jorissen, A., et al. 2017, A\&A, 608, A100

Gorlova, N., Van Winckel, H., Vos, J., et al. 2013, EAS Pub. Ser., 64, 163

Gray, R. O., McGahee, C. E., Griffin, R. E. M., \& Corbally, C. J. 2011, AJ, 141, 160

Griffin, R. F. 2018, The Observatory, 138, 10

Gustafsson, B., Edvardsson, B., Eriksson, K., et al. 2008, A\&A, 486, 951

Henry, T. J., Soderblom, D. R., Donahue, R. A., \& Baliunas, S. L. 1996, AJ, 111, 00

Herwig, F., Bloecker, T., Schoenberner, D., \& El Eid, M. 1997, A\&A, 324, L81

Houk, N. 1978, University of Michigan Catalogue of Two-dimensional Spectral Types for the HD Stars (Ann Arbor: University of Michigan), II

Houk, N., \& Cowley, A. P. 1975, University of Michigan Catalogue of Twodimensional Spectral Types for the HD Stars (Ann Arbor: University of Michigan), I

Izzard, R. G., Dermine, T., \& Church, R. P. 2010, A\&A, 523, A10

Jancart, S., Jorissen, A., Babusiaux, C., \& Pourbaix, D. 2005, A\&A, 442, 365

Jorissen, A., \& Boffin, H. M. J. 1992, in Binaries as Tracers of Star Formationx, eds. A. Duquennoy, \& M. Mayor (Cambridge Univ. Press), 110

Jorissen, A., \& Mayor, M. 1988, A\&A, 198, 187

Jorissen, A., Van Eck, S., Mayor, M., \& Udry, S. 1998, A\&A, 332, 877

Jorissen, A., Van Eck, S., Van Winckel, H., et al. 2016, A\&A, 586, A158
Jorissen, A., Boffin, H. M. J., Karinkuzhi, D., et al. 2019, A\&A, 626, A127

Karinkuzhi, D., \& Goswami, A. 2014, MNRAS, 440, 1095

Karinkuzhi, D., Van Eck, S., Jorissen, A., et al. 2018, A\&A, 618, A32

Keenan, P. C. 1942, ApJ, 96, 101

Kleinman, S. J., Kepler, S. O., Koester, D., et al. 2013, ApJS, 204, 5

Kniazev, A. Y., Gvaramadze, V. V., \& Berdnikov, L. N. 2016, MNRAS, 459, 3068

Kordopatis, G., Gilmore, G., Steinmetz, M., et al. 2013, AJ, 146, 134

Kurtz, M. J., \& Mink, D. J. 1998, PASP, 110, 934

Lindegren, L., Hernández, J., Bombrun, A., et al. 2018, A\&A, 616, A2

Lü, P. K. 1991, AJ, 101, 2229

Lü, P. K., Dawson, D. W., Upgren, A. R., \& Weis, E. W. 1983, ApJS, 52, 169

Luck, R. E., \& Bond, H. E. 1991, ApJS, 77, 515

Lucy, L. B., \& Sweeney, M. A. 1971, AJ, 76, 544

Manick, R., Van Winckel, H., Kamath, D., Hillen, M., \& Escorza, A. 2017, A\&A, 597, A129

Masseron, T., Merle, T., \& Hawkins, K. 2016, Astrophysics Source Code Library [record ascl:1205.004]

Mathieu, R. D., \& Geller, A. M. 2015, in The Blue Stragglers of the Old Open Cluster NGC 188, eds. H. M. J. Boffin, G. Carraro, \& G. Beccari, 29

McClure, R. D. 1984, PASP, 96, 117

McClure, R. D., \& Woodsworth, A. W. 1990, ApJ, 352, 709

Merle, T., Jorissen, A., Van Eck, S., Masseron, T., \& Van Winckel, H. 2016, A\&A, 586, A151

Mikołajewska, J. 2012, Balt. Astron., 21, 5

Miszalski, B., Manick, R., Mikołajewska, J., et al. 2018a, MNRAS, 473, 2275

Miszalski, B., Manick, R., Mikołajewska, J., Van Winckel, H., \& Iłkiewicz, K. 2018b, PASA, 35, e027

Murphy, S. J., Moe, M., Kurtz, D. W., et al. 2018, MNRAS, 474, 4322

North, P. 1995, Mem. Soc. Astron. It., 66, 379

North, P., \& Duquennoy, A. 1991, A\&A, 244, 335

North, P., \& Lanz, T. 1991, A\&A, 251, 489

North, P., Berthet, S., \& Lanz, T. 1994, A\&A, 281, 775

North, P., Jorissen, A., \& Mayor, M. 2000, IAU Symp., 177, 269

O'Donoghue, D., Buckley, D. A. H., Balona, L. A., et al. 2006, MNRAS, 372, 151

Oomen, G.-M., Van Winckel, H., Pols, O., et al. 2018, A\&A, 620, A85

Pereira, C. B., \& Junqueira, S. 2003, A\&A, 402, 1061

Perryman, M. A. C., Lindegren, L., Kovalevsky, J., et al. 1997, A\&A, 500, 501

Plez, B. 2012, Astrophysics Source Code Library [record ascl:1205.004]

Pols, O. R., Karakas, A. I., Lattanzio, J. C., \& Tout, C. A. 2003, ASP Conf. Ser., 303, 290

Pourbaix, D., \& Arenou, F. 2001, A\&A, 372, 935

Pourbaix, D., \& Boffin, H. M. J. 2003, A\&A, 398, 1163

Pourbaix, D. \& Jorissen, A. 2000, A\&AS, 145, 161

Pourbaix, D., Tokovinin, A. A., Batten, A. H., et al. 2004, A\&A, 424, 727

Raskin, G., \& Van Winckel, H. 2014, Astron. Nachr., 335, 32

Raskin, G., van Winckel, H., Hensberge, H., et al. 2011, A\&A, 526, A69

Schröder, K.-P., \& Cuntz, M. 2007, A\&A, 465, 593

Siess, L., \& Arnould, M. 2008, A\&A, 489, 395

Siess, L., Dufour, E., \& Forestini, M. 2000, A\&A, 358, 593

Siess, L., Izzard, R. G., Davis, P. J., \& Deschamps, R. 2013, A\&A, 550, A100

Soderblom, D. R., King, J. R., \& Henry, T. J. 1998, AJ, 116, 396

Soubiran, C., Jasniewicz, G., Chemin, L., et al. 2013, A\&A, 552, A64

Stahl, O., Kaufer, A., \& Tubbesing, S. 1999, ASP Conf. Ser., 188, 331

Tokovinin, A. 2014, AJ, 147, 87

Tokovinin, A., Thomas, S., Sterzik, M., \& Udry, S. 2006, A\&A, 450, 681

Tomkin, J., Lambert, D. L., Edvardsson, B., Gustafsson, B., \& Nissen, P. E. 1989, A\&A, 219, L15

Toonen, S., Hamers, A., \& Portegies Zwart, S. 2016, Comput. Astrophys. Cosmol., 3, 6

Torres, G., \& Stefanik, R. P. 2000, AJ, 119, 1914

Udry, S., Jorissen, A., Mayor, M., \& Van Eck, S. 1998a, A\&AS, 131, 25

Udry, S., Mayor, M., Van Eck, S., et al. 1998b, A\&AS, 131, 43

Udry, S., Mayor, M., \& Queloz, D. 1999, ASP Conf. Ser., 185, 367

Van Winckel, H., Jorissen, A., Gorlova, N., et al. 2010, MemSAI, 81, 1022

Vanture, A. D. 1992a, AJ, 103, 2035

Vanture, A. D. 1992b, AJ, 104, 1986

Vanture, A. D. 1992c, AJ, 104, 1997

Vos, J., Østensen, R. H., Marchant, P., \& Van Winckel, H. 2015, A\&A, 579, A49

Vos, J., Østensen, R. H., Vučković, M., \& Van Winckel, H. 2017, A\&A, 605, A109

Wallerstein, G., \& Knapp, G. R. 1998, ARA\&A, 36, 369

Webbink, R. F. 1986, Highlights Astron., 7, 185

Willmarth, D. W., Fekel, F. C., Abt, H. A., \& Pourbaix, D. 2016, AJ, 152, 46

Yamashita, Y. 1975, PASJ, 27, 325 


\section{Appendix A: Sample}

Table A.1. Information about the initial sample considered for this paper including classification of the objects in the literature (Col. 3), number of available data points from each instrument (Cols. 4-7), and binarity status after this work (Col. 8).

\begin{tabular}{|c|c|c|c|c|c|c|c|}
\hline ID used in this paper & Second ID & Type (Ref.) & CORAVEL & HERMES & SALT HRS & OTHER & Binarity \\
\hline BD- $10^{\circ} 4311$ & HIP 80356 & $\operatorname{sgCH}(1)$ & 29 & 20 & 0 & 1 & SB-O \\
\hline $\mathrm{BD}-11^{\circ} 3853$ & HIP 73444 & $\operatorname{sgCH}(1)$ & 16 & 113 & 0 & 2 & SB \\
\hline $\mathrm{BD}-18^{\circ} 255$ & TYC 5852-1110-1 & $\mathrm{dBa}(2,3,4), \operatorname{sgCH}(1)$ & 14 & 36 & 0 & 0 & SB-O \\
\hline $\mathrm{BD}+18^{\circ} 5215$ & TYC 1724-1717-1 & $\mathrm{dBa}(5)$ & 28 & 3 & 0 & 0 & SB-O \\
\hline $\mathrm{CD}-62^{\circ} 1346$ & HIP 104151 & $\operatorname{sgCH}(1)$ & 4 & 0 & 8 & 0 & SB \\
\hline HD 2454 & $\mathrm{BD}+09^{\circ} 47$ & $\mathrm{dBa}(6)$ & 46 & 54 & 0 & 0 & SB \\
\hline HD 6434 & HIP 5054 & $\mathrm{dBa}(7)$ & 11 & 0 & 1 & 0 & NV \\
\hline HD 9529 & $\mathrm{CD}-71^{\circ} 71$ & $\mathrm{dBa}(2,3)$ & 7 & 0 & 6 & 0 & NV \\
\hline HD 13555 & $\mathrm{BD}+20^{\circ} 348$ & $\mathrm{dBa}(7)$ & 8 & 52 & 0 & 0 & $\mathrm{NV}$ \\
\hline HD 15306 & $\mathrm{BD}-01^{\circ} 340$ & $\mathrm{dBa}(5)$ & 26 & 28 & 0 & 3 & SB-O \\
\hline HD 18853 & HIP 13842 & $\mathrm{dBa}(2)$ & 3 & 0 & 8 & 5 & SB \\
\hline HD 22589 & $\mathrm{BD}-07^{\circ} 642$ & $\operatorname{gBa}(8,9)$ & 20 & 30 & 0 & 0 & SB-O \\
\hline HD 24864 & $\mathrm{CD}-54^{\circ} 750$ & $\mathrm{dBa}(2)$ & 10 & 0 & 3 & 0 & SB-O \\
\hline HD 26455 & $\mathrm{CD}-53^{\circ} 858$ & $\mathrm{dBa}(2)$ & 1 & 0 & 4 & 5 & SB2 \\
\hline HD 31732 & HIP 22814 & $\mathrm{dBa}(2)$ & 4 & 0 & 2 & 0 & NV \\
\hline HD 34654 & HIP 25222 & $\mathrm{dBa}(10)$ & 0 & 42 & 0 & 0 & SB-O \\
\hline HD 35296 & $\mathrm{BD}+17^{\circ} 920$ & $\mathrm{dBa}(7)$ & 16 & 58 & 0 & 0 & NV \\
\hline HD 48565 & $\mathrm{BD}+20^{\circ} 1552$ & $\mathrm{dBa}(5)$ & 30 & 76 & 0 & 4 & TS \\
\hline HD 50264 & HIP 32894 & $\mathrm{dBa}(8), \operatorname{sgCH}(11)$ & 9 & 1 & 1 & 0 & SB-O \\
\hline HD 60532 & $\mathrm{BD}-21^{\circ} 2007$ & $\mathrm{dBa}(7)$ & 6 & 40 & 0 & 0 & NV \\
\hline HD 69578 & HIP 40208 & $\mathrm{dBa}(2)$ & 8 & 0 & 3 & 0 & SB \\
\hline HD 76225 & HIP 43703 & $\mathrm{dBa}(5)$ & 3 & 22 & 0 & 4 & SB-O \\
\hline HD 82328 & $\mathrm{BD}+52^{\circ} 1401$ & $\mathrm{dBa}(7)$ & 5 & 79 & 0 & 0 & NV \\
\hline HD 87080 & HIP 49166 & $\mathrm{dBa}(8), \operatorname{sgCH}(11)$ & 9 & 1 & 0 & 0 & SB-O \\
\hline HD 89948 & HIP 50805 & $\operatorname{sgCH}(1)$ & 18 & 1 & 0 & 0 & SB-O \\
\hline HD 92545 & $\mathrm{BD}-11^{\circ} 2929$ & $\mathrm{dBa}(5)$ & 19 & 72 & 0 & 0 & SB \\
\hline HD 95241 & $\mathrm{BD}+43^{\circ} 2068$ & $\mathrm{dBa}(7)$ & 9 & 61 & 0 & 0 & SB-O \\
\hline HD 98991 & $\mathrm{BD}-17^{\circ} 3367$ & $\mathrm{dBa}(7)$ & 8 & 39 & 0 & 0 & SB-O \\
\hline HD 101581 & HIP 56998 & suspected $\mathrm{dBa}(12)$ & 9 & 0 & 0 & 0 & NV \\
\hline HD 103840 & HIP 58290 & suspected $\mathrm{dBa}(12)$ & 10 & 0 & 0 & 0 & NV \\
\hline HD 104342 & HIP 58582 & suspected $\mathrm{dBa}(12)$ & 7 & 0 & 0 & 0 & NV \\
\hline HD 105671 & HIP 59296 & suspected dBa(12) & 6 & 0 & 0 & 0 & $\mathrm{NV}$ \\
\hline HD 106191 & BD-14운 3478 & $\mathrm{dBa}(5)$ & 29 & 5 & 0 & 0 & SB-O \\
\hline HD 107574 & HIP 60299 & $\mathrm{dBa}(5)$ & 21 & 55 & 0 & 0 & SB-O \\
\hline HD 109490 & $\mathrm{CD}-43^{\circ} 7765$ & $\mathrm{dBa}(3)$ & 3 & 0 & 0 & 0 & SB \\
\hline HD 113402 & HIP 63812 & $\mathrm{dBa}(5)$ & 8 & 0 & 0 & 0 & $\mathrm{NV}$ \\
\hline HD 114520 & $\mathrm{BD}+22^{\circ} 2550$ & $\mathrm{dBa}(10)$ & 0 & 85 & 0 & 0 & SB2 \\
\hline HD 117288 & HIP 65870 & suspected $\mathrm{dBa}(12)$ & 9 & 0 & 0 & 0 & NV \\
\hline HD 120620 & $\mathrm{BD}-03^{\circ} 3537$ & $\mathrm{gBa}(8,9)$ & 28 & 3 & 0 & 0 & SB-O \\
\hline HD 123585 & HIP 69176 & $\operatorname{sgCH}(1)$ & 11 & 0 & 0 & 0 & SB-O \\
\hline HD 124850 & $\mathrm{BD}-05^{\circ} 3843$ & $\mathrm{dBa}(7)$ & 18 & 86 & 0 & 0 & NV \\
\hline HD 127392 & HIP 71058 & $\operatorname{sgCH}(1)$ & 10 & 1 & 0 & 0 & SB-O \\
\hline
\end{tabular}

Notes. Column 7 "OTHER" lists the number of available CORALIE radial-velocity measurements for all targets except HD 48565, for which we have four ELODIE radial-velocity points, but no CORALIE data. Binarity type in Col. 8: SB-O: spectroscopic binary with determined orbital parameters in Table 1; SB: confirmed spectroscopic binary without available orbit (see Table 2); SB2: binary with a double-peaked CCF; TS: confirmed triple system; NV: no variability detected according to the criterion described in Sect. 4.5.

References. For Col. 3: 1: Luck \& Bond (1991); 2: Houk \& Cowley (1975); 3: Houk (1978); 4: North (1995); 5: North et al. (1994); 6: Tomkin et al. (1989); 7: Edvardsson et al. (1993); 8: Lü et al. (1983); 9: Udry et al. (1998a); 10: Gray et al. (2011); 11: (Pereira \& Junqueira 2003); 12: Lü (1991); 13: McClure \& Woodsworth (1990). 
Table A.1. continued.

\begin{tabular}{|c|c|c|c|c|c|c|c|}
\hline ID used in this paper & Second ID & Type (Ref.) & CORAVEL & HERMES & SALT HRS & OTHER & Binarity \\
\hline HD 130255 & $\mathrm{BD}+01^{\circ} 2980$ & $\operatorname{sgCH}(13)$ & 26 & 68 & 0 & 0 & NV \\
\hline HD 141804 & $\mathrm{CD}-53^{\circ} 6286$ & $\operatorname{sgCH}(1)$ & 8 & 0 & 0 & 0 & SB-O \\
\hline HD 146800 & HIP 80043 & suspected $\mathrm{dBa}(12)$ & 9 & 0 & 0 & 0 & NV \\
\hline HD 147609 & $\mathrm{BD}+27^{\circ} 2631$ & $\mathrm{dBa}(5)$ & 21 & 41 & 0 & 0 & SB-O \\
\hline HD 150862 & $\mathrm{CD}-24^{\circ} 12805$ & $\operatorname{sgCH}(1)$ & 12 & 1 & 0 & 0 & SB-O \\
\hline HD 170149 & HIP 90674 & suspected $\mathrm{dBa}(12)$ & 8 & 0 & 2 & 0 & NV \\
\hline HD 177996 & HIP 94050 & suspected $\mathrm{dBa}(12)$ & 16 & 0 & 2 & 0 & SB2 \\
\hline HD 182274 & $\mathrm{BD}+19^{\circ} 3996$ & $\operatorname{sgCH}(1)$ & 19 & 11 & 0 & 0 & SB-O \\
\hline HD 188985 & HIP 98431 & $\mathrm{dBa}(5)$ & 2 & 0 & 2 & 0 & SB \\
\hline HD 202400 & HIP 105294 & $\mathrm{dBa}(5)$ & 5 & 0 & 9 & 0 & NV \\
\hline HD 205156 & HIP 106560 & suspected $\mathrm{dBa}(12)$ & 17 & 0 & 2 & 0 & SB \\
\hline HD 207585 & HIP 107818 & $\operatorname{sgCH}(1)$ & 16 & 2 & 2 & 0 & SB-O \\
\hline HD 216219 & $\mathrm{BD}+17^{\circ} 4818$ & $\operatorname{gBa}(8,9,12)$ & 29 & 1 & 0 & 0 & SB-O \\
\hline HD 219899 & HIP 115183 & suspected $\mathrm{dBa}(12)$ & 8 & 0 & 0 & 0 & NV \\
\hline HD 220117 & $\mathrm{BD}+37^{\circ} 4817$ & $\mathrm{dBa}(7)$ & 11 & 69 & 0 & 0 & NV \\
\hline HD 221531 & $\mathrm{BD}-12^{\circ} 6514$ & $\mathrm{dBa}(5)$ & 29 & 40 & 0 & 0 & SB-O \\
\hline HD 222349 & $\mathrm{CD}-57^{\circ} 8842$ & $\mathrm{dBa}(5)$ & 4 & 0 & 4 & 1 & SB \\
\hline HD 224621 & HIP 118266 & $\operatorname{sgCH}(1)$ & 4 & 0 & 0 & 0 & SB \\
\hline
\end{tabular}

\section{Appendix B: Orbital solutions of the Ba dwarfs}

Figures from B.1 to B.25 show the RV curves and the bestfitting solutions of the 25 new orbits obtained for this paper. The upper panel of each graph includes, when available for each specific target, HERMES (black dots), CORAVEL (orange stars), CORALIE (purple triangles), and SALT (blue squares) radial velocity data, and the best-fitting model of the orbit (black solid line). The lower panel includes the $O-C$ residuals of the fit and a shadowed region which corresponds to three times the standard deviation of the residuals.

Figure B.26 shows the available data for the objects that, according to the criterion explained and applied in Sect. 4.5, are in binary systems even though we cannot constrain their orbital element. Finally, Fig. B.27 collects the RV data of all the remaining objects. For some of them, we simply do not have enough data to be conclusive, while others are probably not binaries.

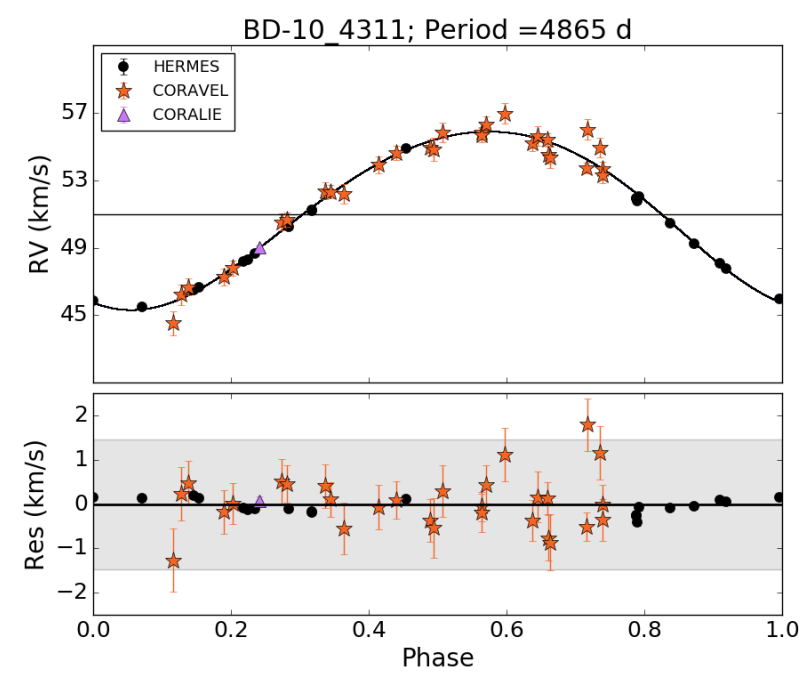

Fig. B.1. BD $-10^{\circ} 4311$.

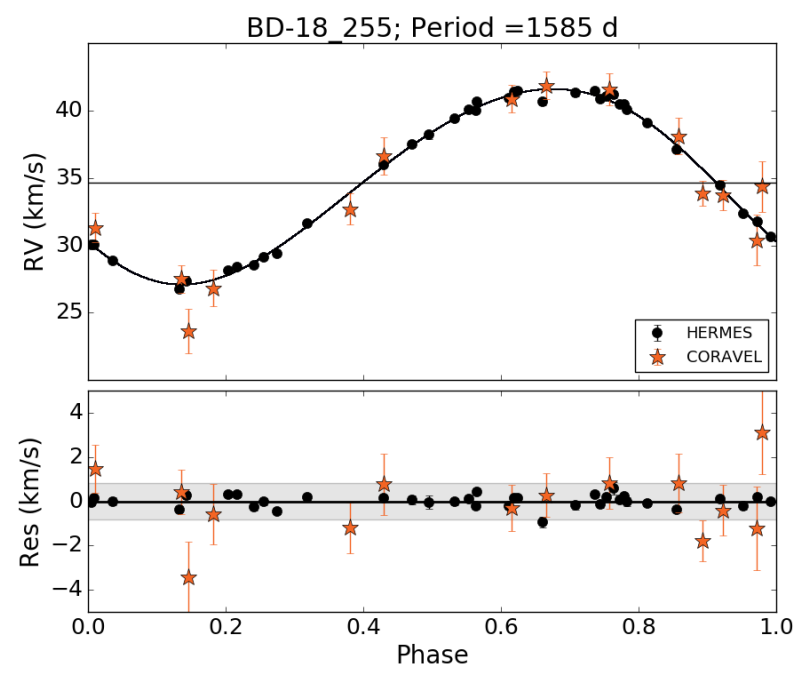

Fig. B.2. $B D-18^{\circ} 255$.

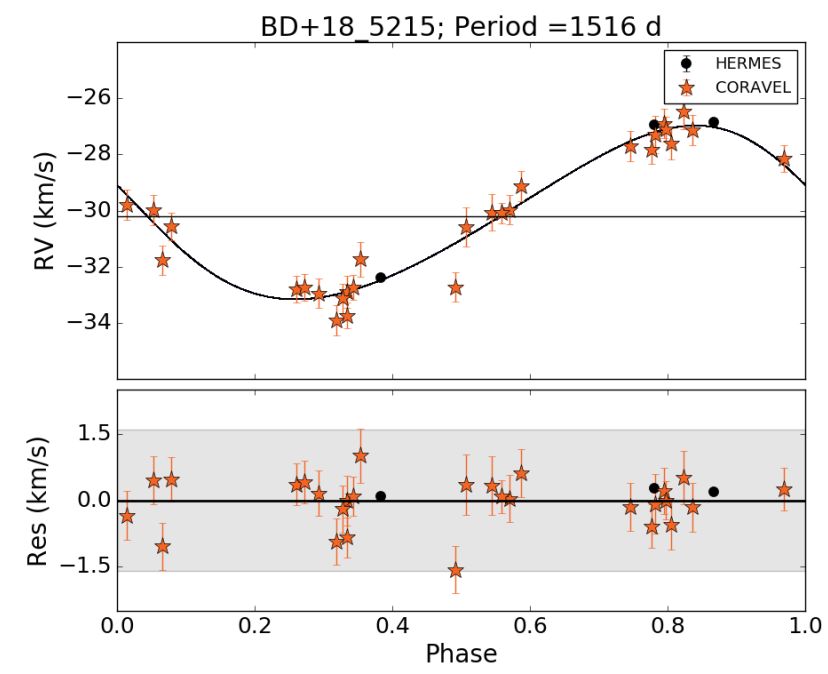

Fig. B.3. $\mathrm{BD}+18^{\circ} 5215$. 


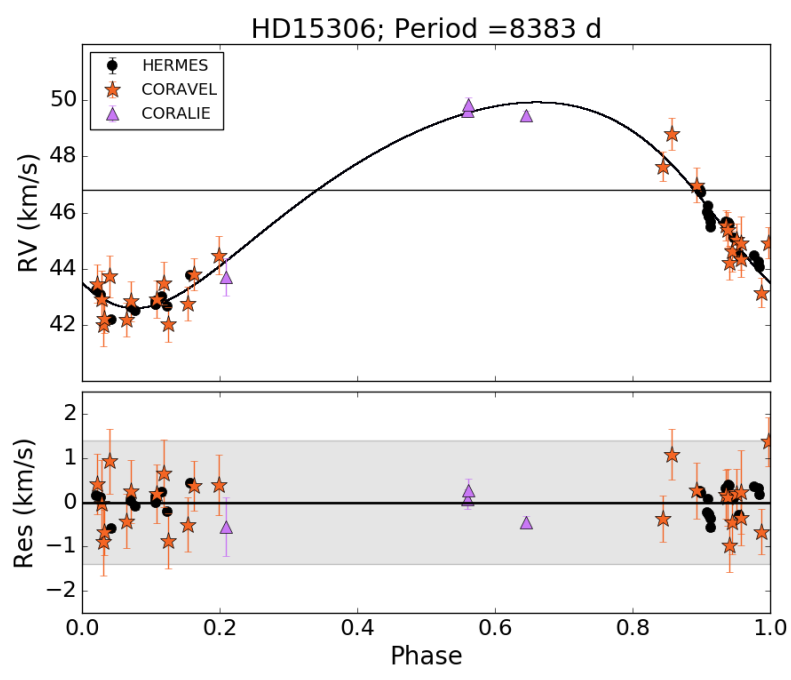

Fig. B.4. HD 15306.

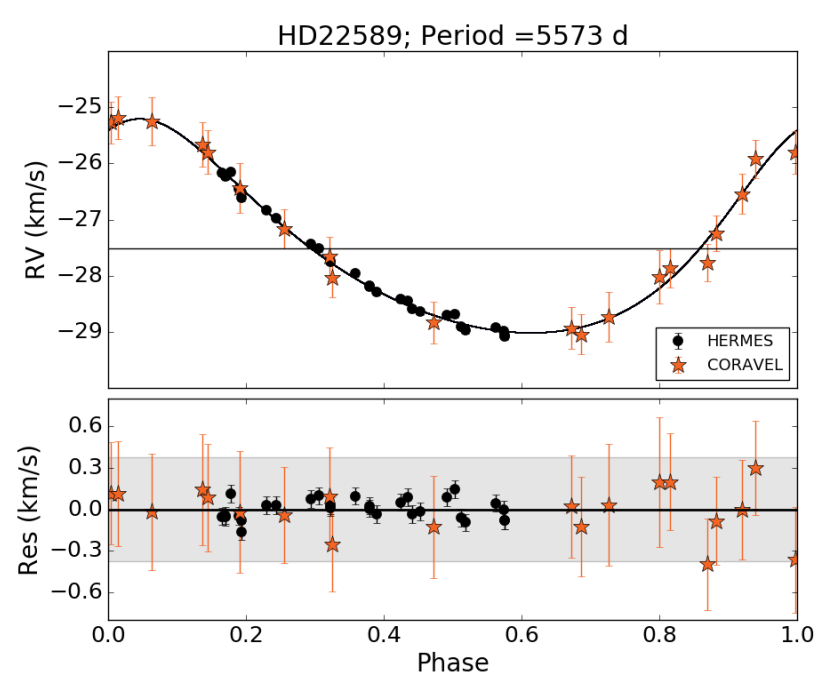

Fig. B.5. HD 22589 .

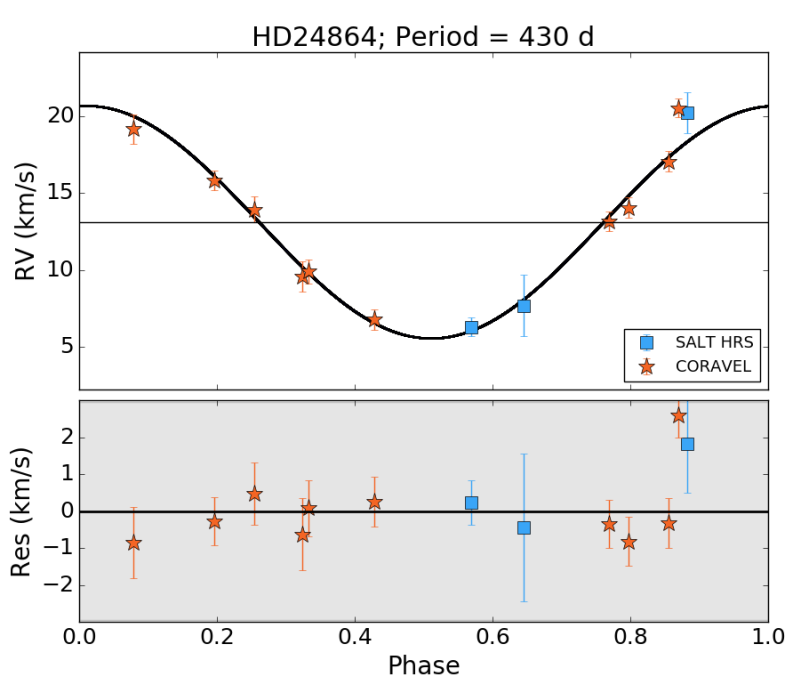

Fig. B.6. HD 24864 .

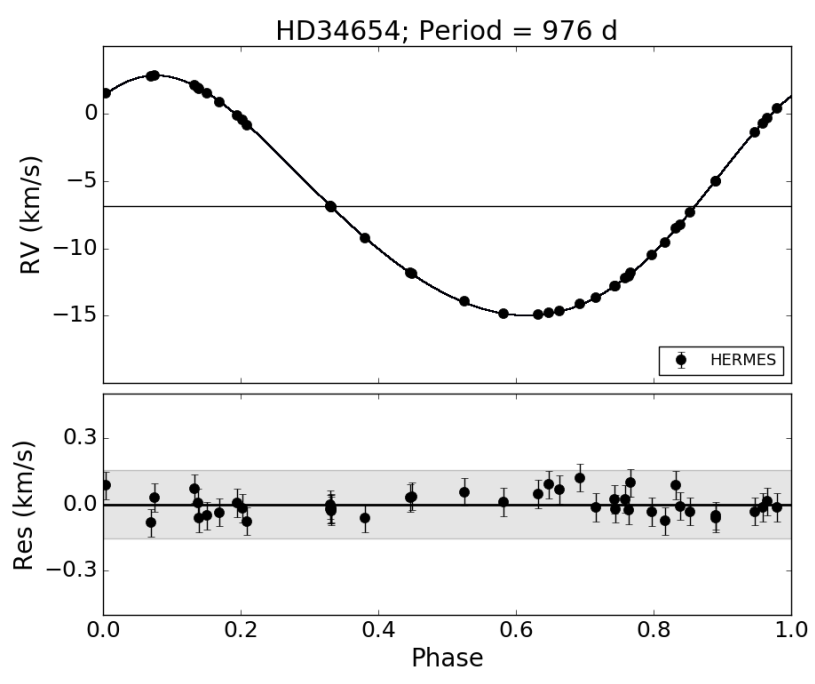

Fig. B.7. HD 34654 .

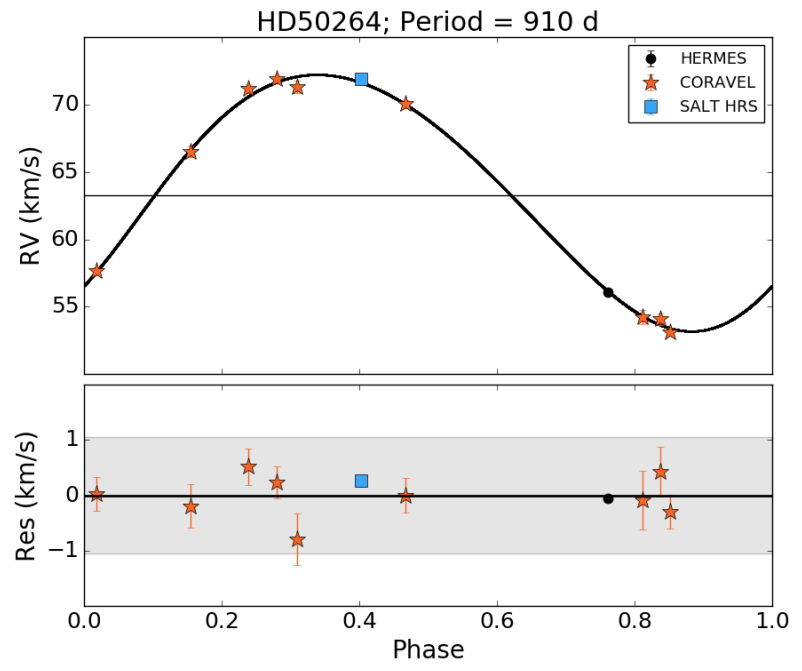

Fig. B.8. HD 50264 .

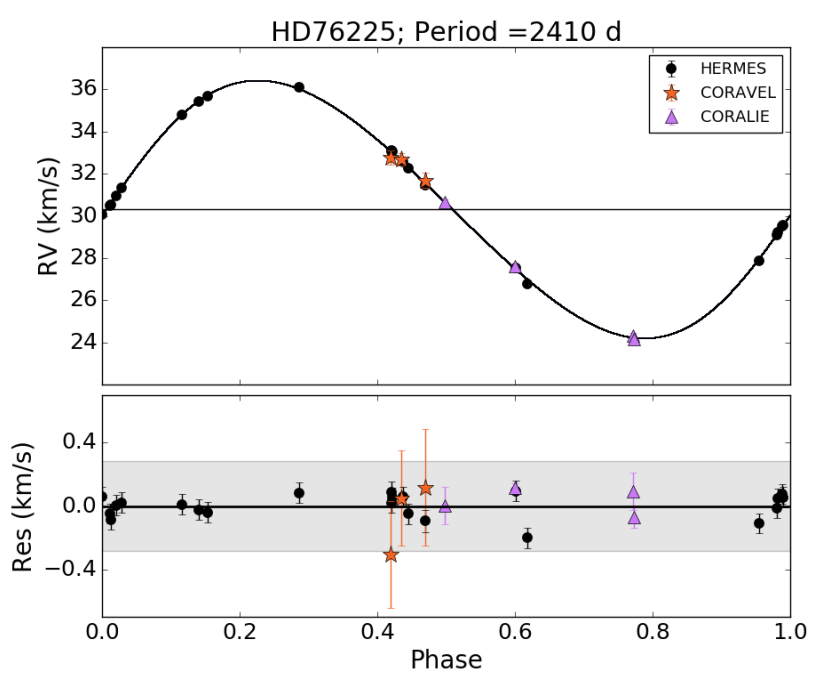

Fig. B.9. HD 76225 . 


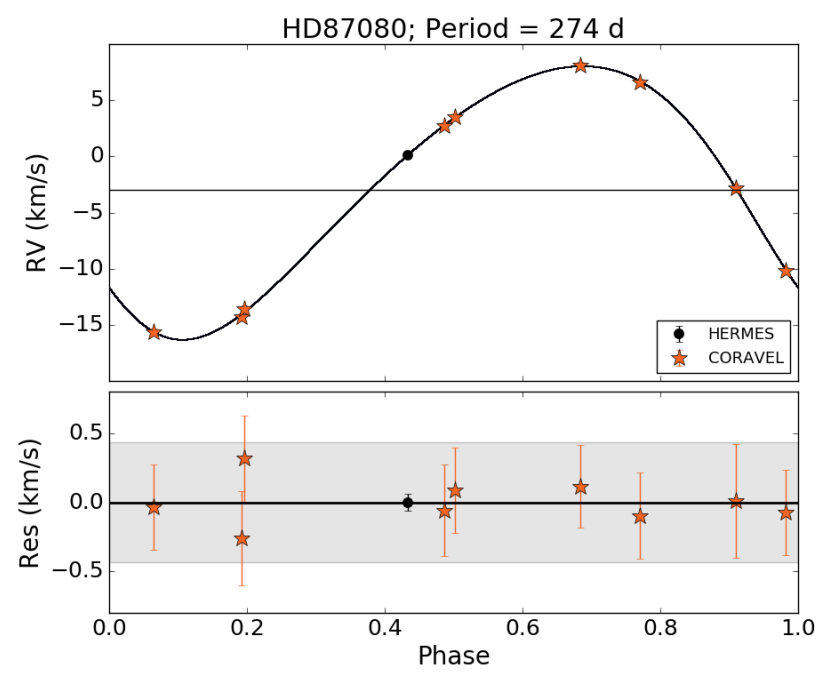

Fig. B.10. HD 87080 .

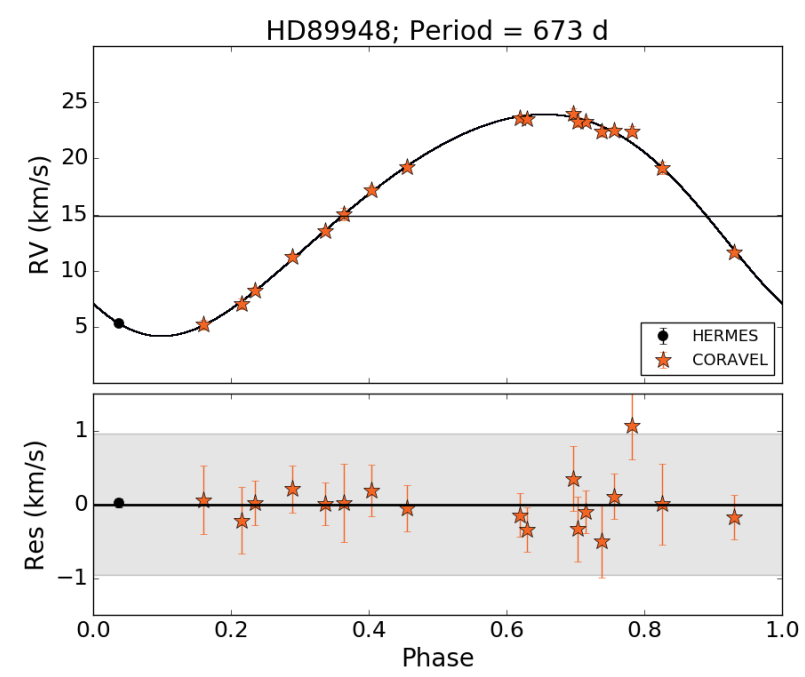

Fig. B.11. HD 89948.

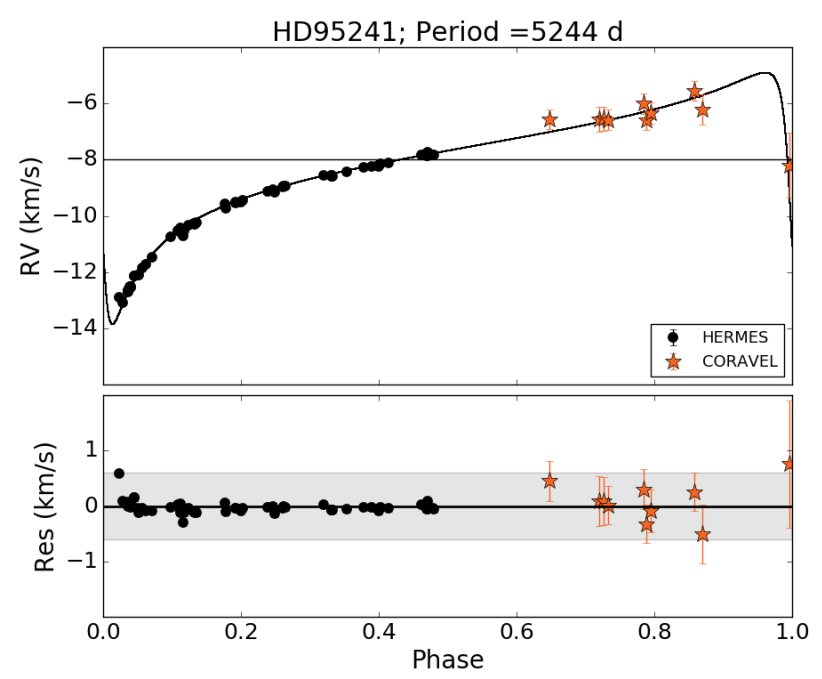

Fig. B.12. HD 95241.

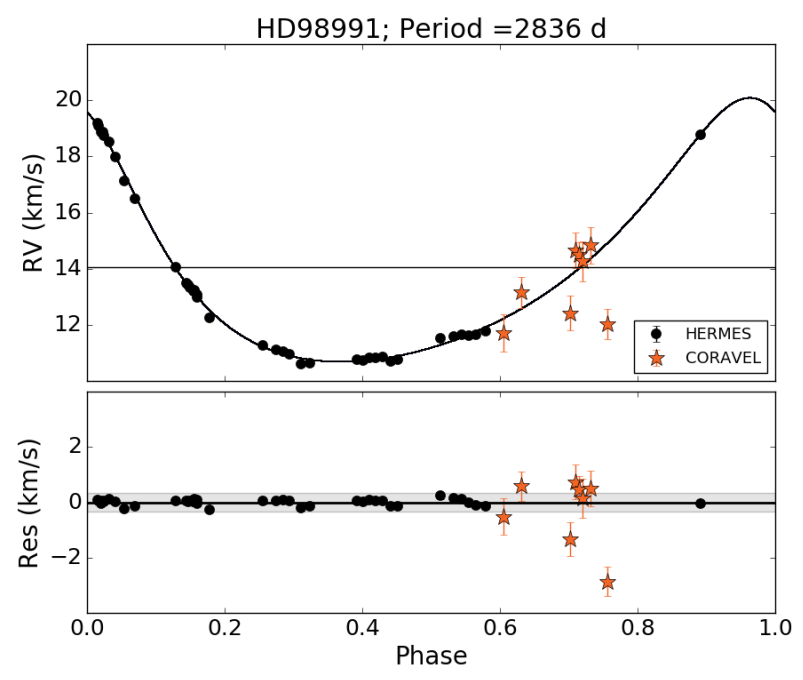

Fig. B.13. HD 98991.

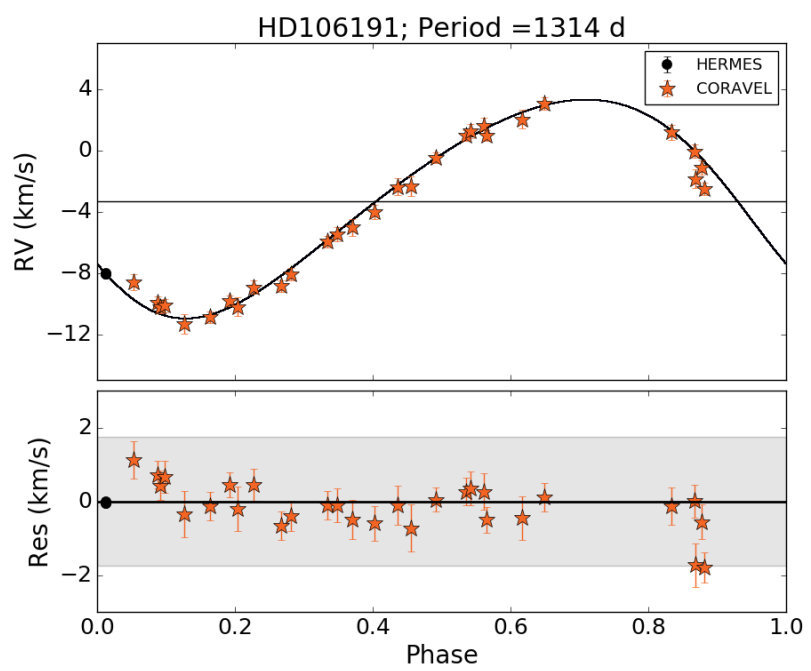

Fig. B.14. HD 106191.

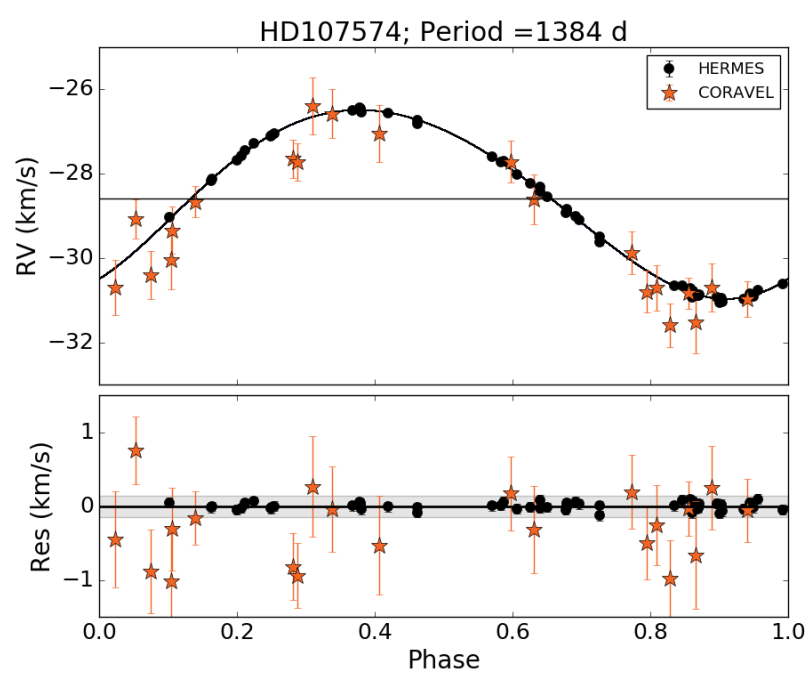

Fig. B.15. HD 107574. 


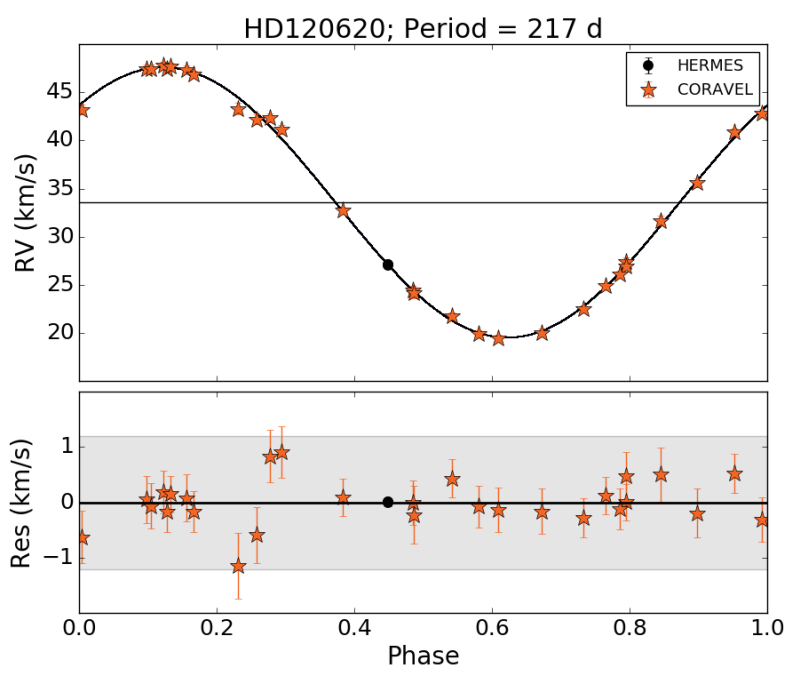

Fig. B.16. HD 120620 .

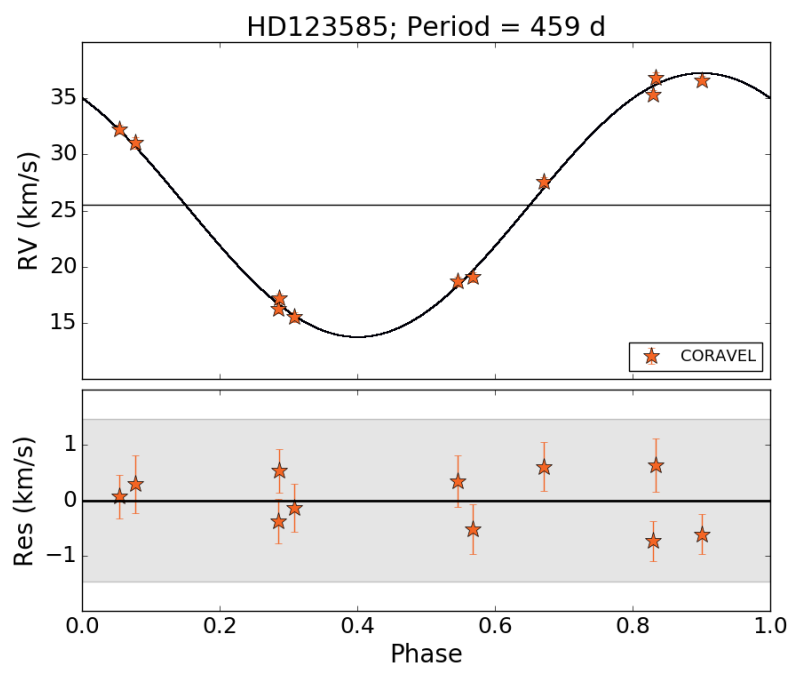

Fig. B.17. HD 123585.

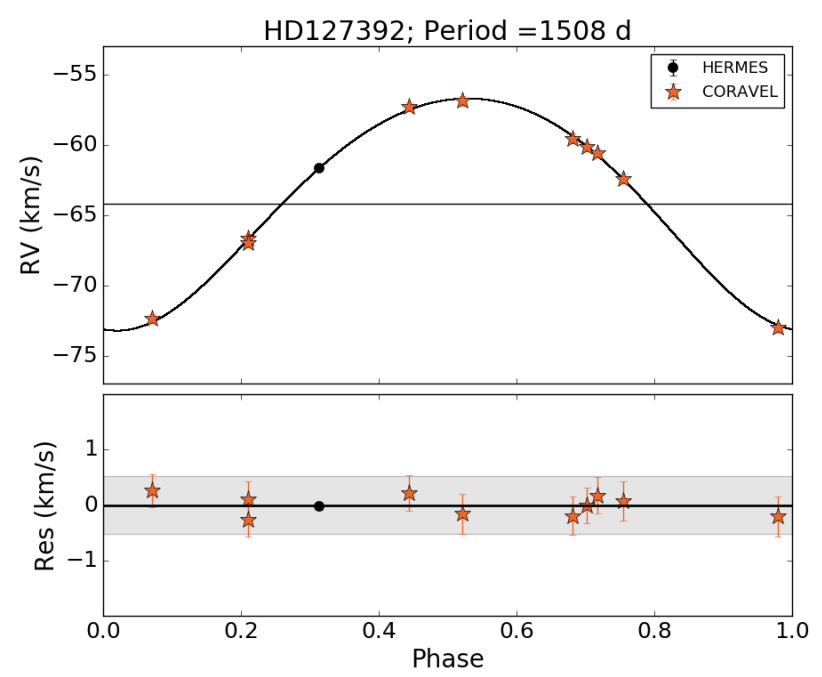

Fig. B.18. HD 127392.

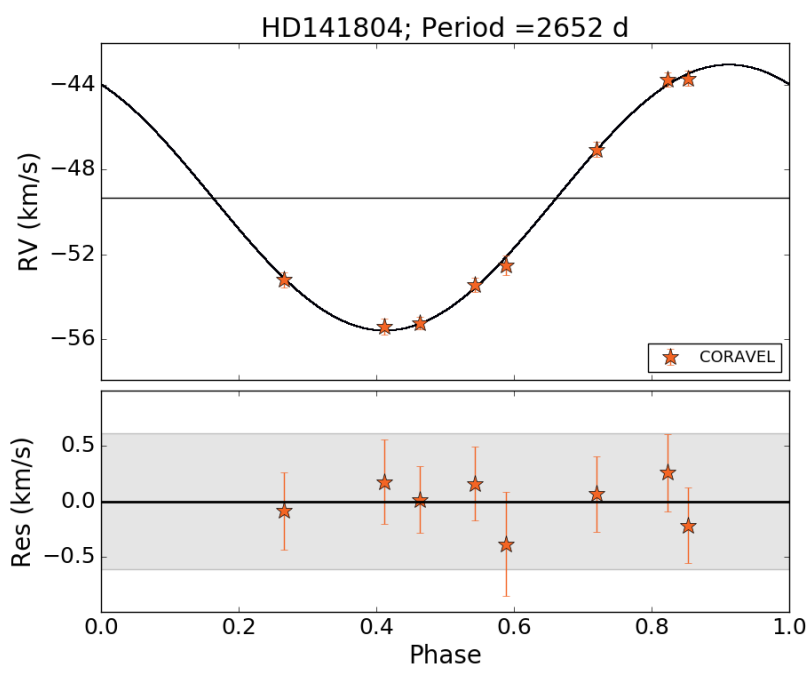

Fig. B.19. HD 141804.

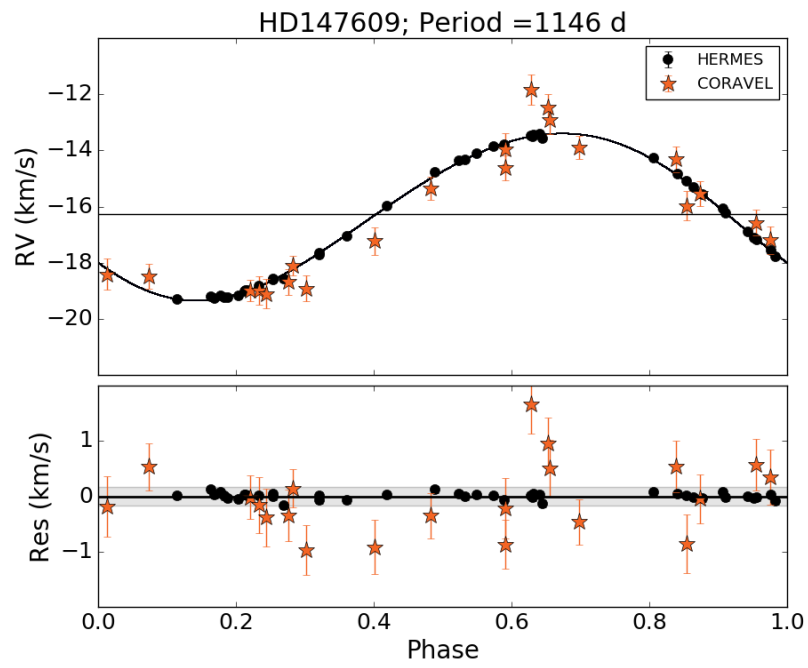

Fig. B.20. HD 147609.

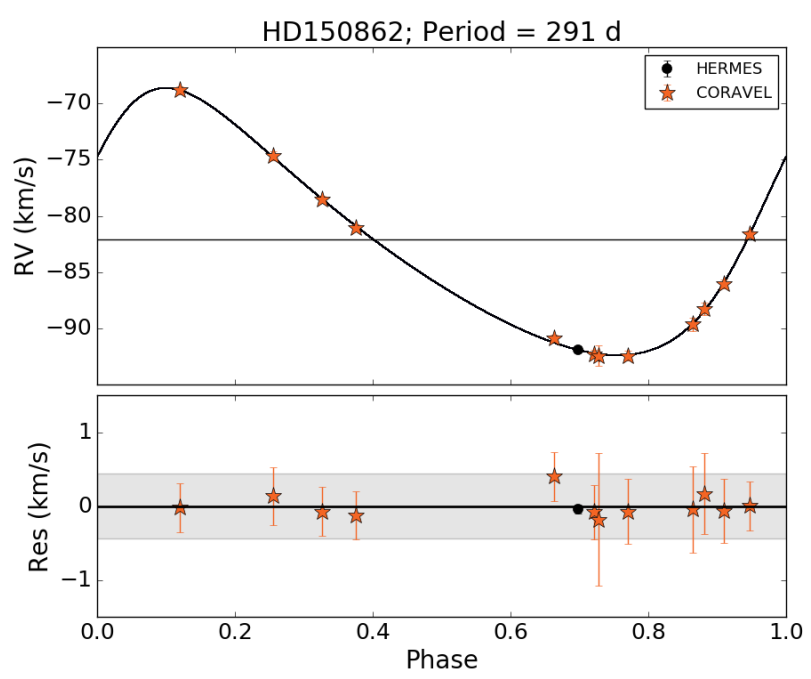

Fig. B.21. HD 150862. 


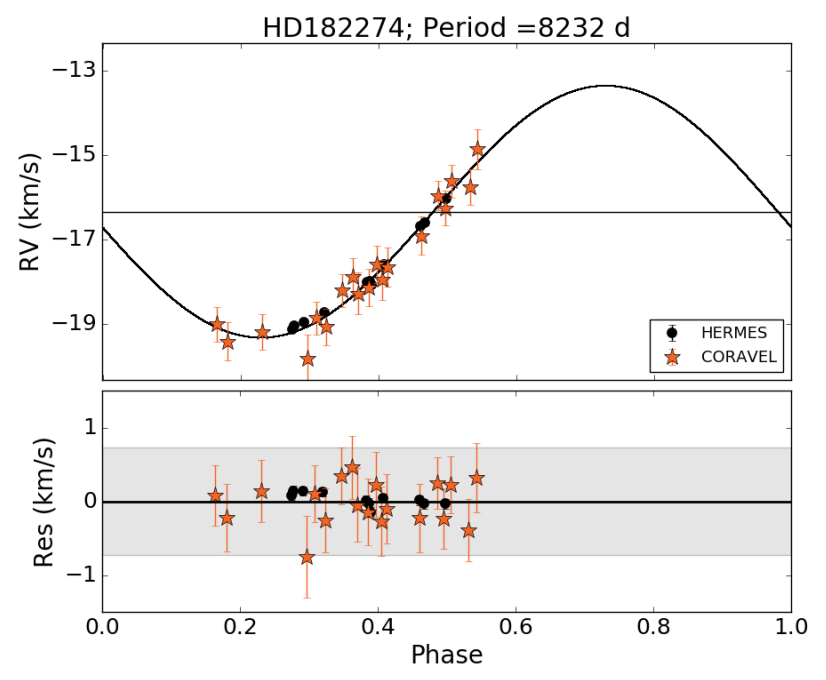

Fig. B.22. HD 182274.

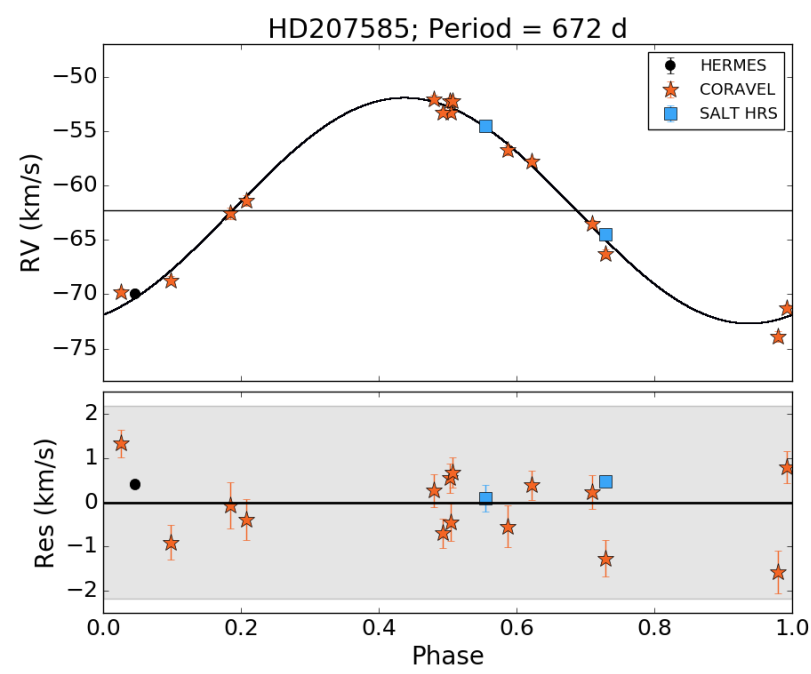

Fig. B.23. HD 207585.

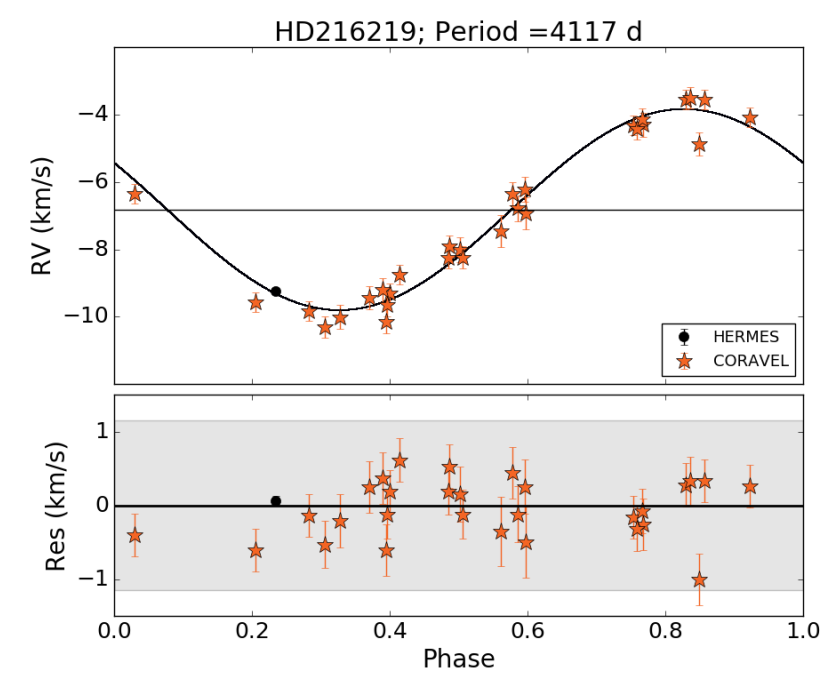

Fig. B.24. HD 216219.

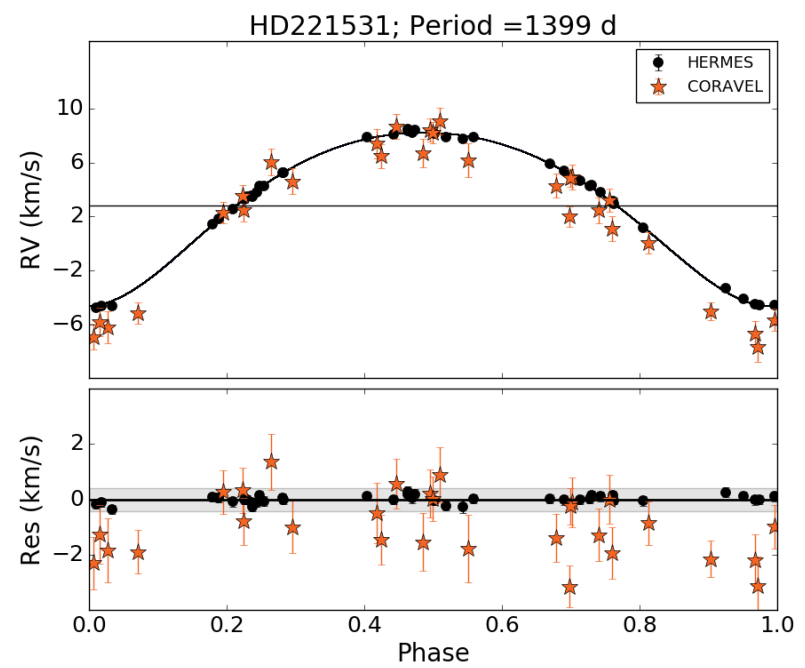

Fig. B.25. HD 221531. 
A. Escorza et al.: Orbits of Ba dwarfs
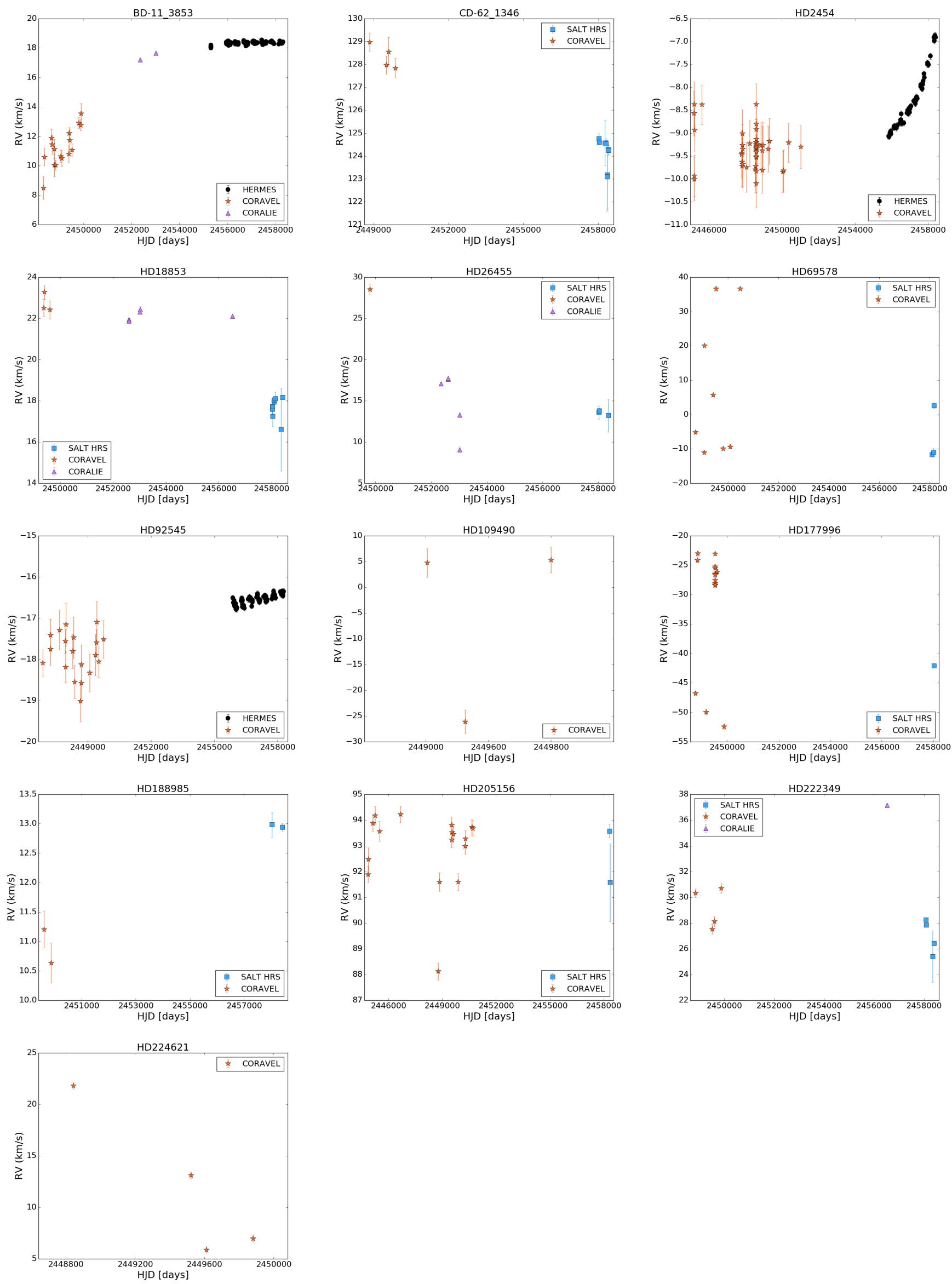

Fig. B.26. HERMES, CORAVEL, CORALIE, and SALT radial velocity data of SBs with no constrained orbit yet available. 
A\&A 626, A128 (2019)
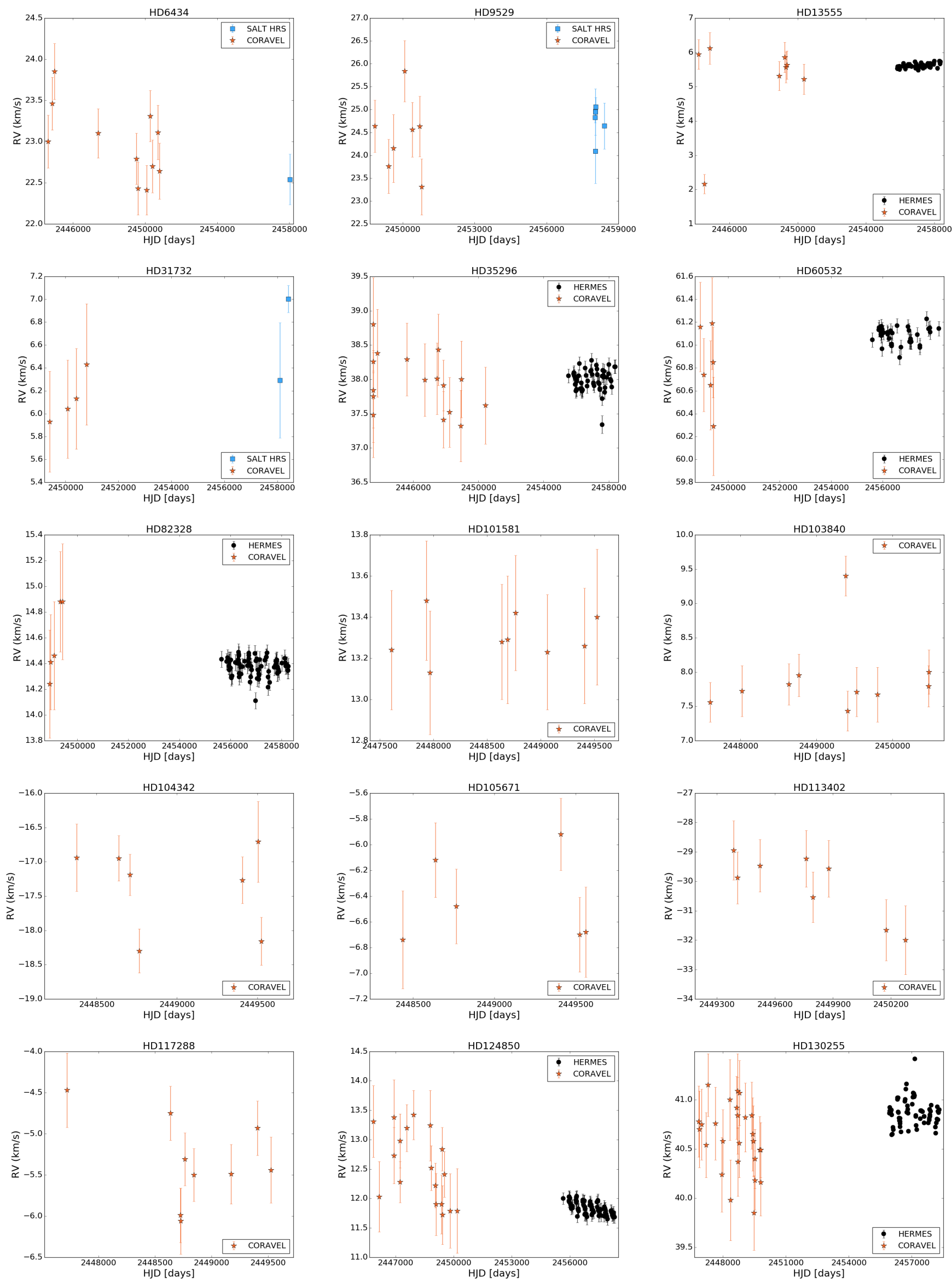

Fig. B.26. continued. 
A. Escorza et al.: Orbits of Ba dwarfs
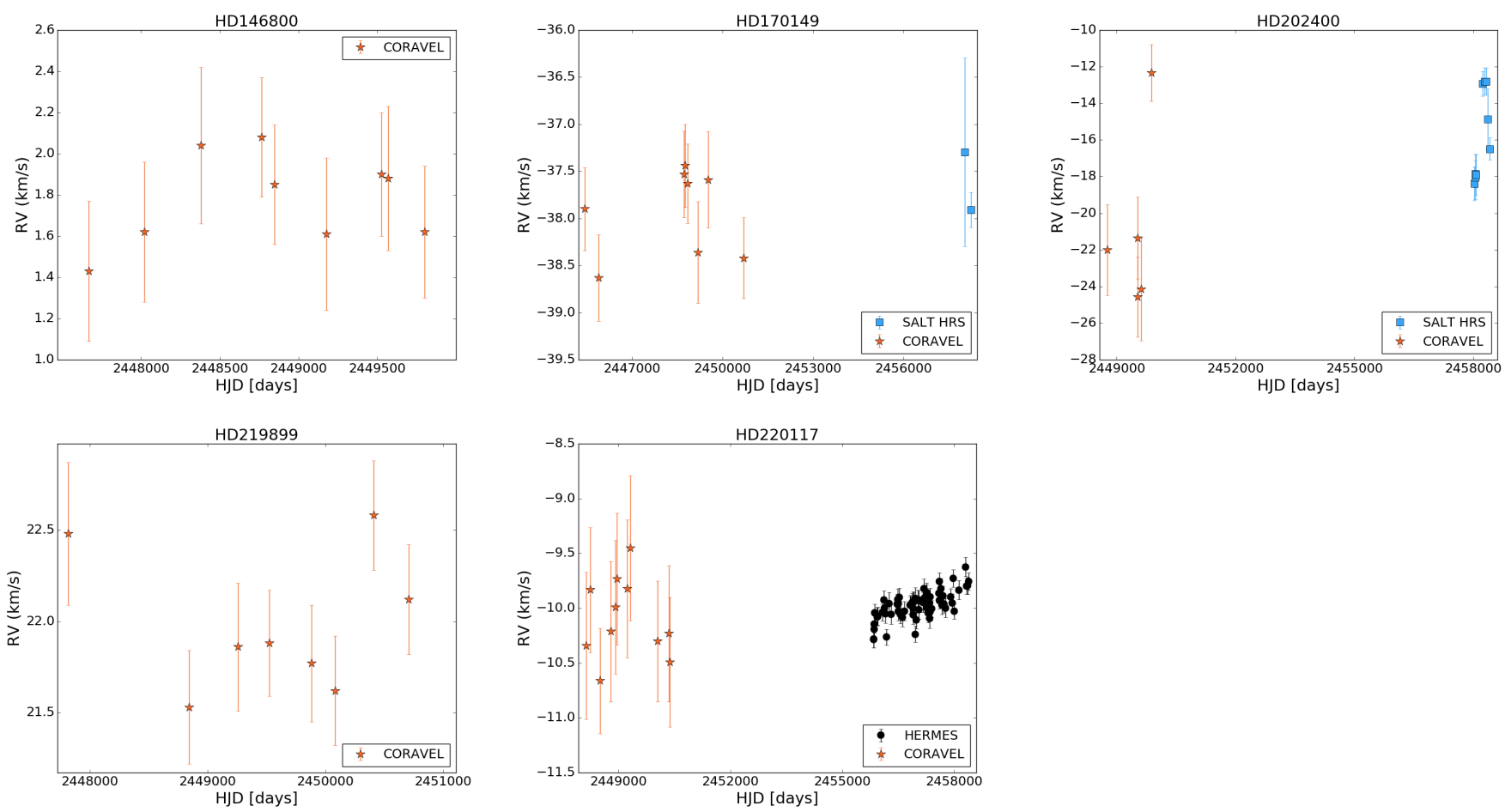

Fig. B.27. HERMES, CORAVEL, CORALIE, and SALT radial velocity data of the targets with non-variable radial velocity data according to our criterion. 